\title{
Topological phases in gated bilayer graphene: Effects of Rashba spin-orbit coupling and exchange field
}

\author{
Zhenhua Qiao, ${ }^{1}$ Xiao Li, ${ }^{1}$ Wang-Kong Tse, ${ }^{1}$ Hua Jiang, ${ }^{2}$ Yugui Yao, ${ }^{3}$ and Qian $\mathrm{Niu}^{1,2}$ \\ ${ }^{1}$ Department of Physics, The University of Texas at Austin, Austin, Texas 78712, USA \\ ${ }^{2}$ International Center for Quantum Materials, Peking University, Beijing 100871, China \\ ${ }^{3}$ School of Physics, Beijing Institute of Technology, Beijing 100081, China
}

(Dated: June 26, 2018)

\begin{abstract}
We present a systematic study on the influence of Rashba spin-orbit coupling, interlayer potential difference and exchange field on the topological properties of bilayer graphene. In the presence of only Rashba spin-orbit coupling and interlayer potential difference, the band gap opening due to broken out-of-plane inversion symmetry offers new possibilities of realizing tunable topological phase transitions by varying an external gate voltage. We find a two-dimensional $Z_{2}$ topological insulator phase and a quantum valley Hall phase in $A B$-stacked bilayer graphene and obtain their effective low-energy Hamiltonians near the Dirac points. For $A A$ stacking, we do not find any topological insulator phase in the presence of large Rashba spin-orbit coupling. When the exchange field is also turned on, the bilayer system exhibits a rich variety of topological phases including a quantum anomalous Hall phase, and we obtain the phase diagram as a function of the Rashba spin-orbit coupling, interlayer potential difference, and exchange field.
\end{abstract}

PACS numbers: 73.22.Pr, 73.43.Cd, 71.70.Ej, 73.43.-f

\section{INTRODUCTION}

Topological insulator $\underline{\underline{1}}$ (TI) is a new phase of quantum matter in materials with strong spin-orbit coupling. The quantum spin Hall effects in graphene ${ }^{2}$ and HgTe quantum wells $\frac{3}{3}$ represent the first examples of twodimensional topological insulators. So far, semiconductor heterostructures like $\mathrm{HgTe}^{\underline{5}}$ and $\mathrm{InAs} / \mathrm{GaSb}^{\underline{6}}$ quantum wells offer the only realistic materials with strong spinorbit coupling that can realize the quantum spin Hall phase, and the intrinsic spin-orbit coupling in graphene was shown to be too weak. $\underline{\underline{4}}$ Due to graphene's attractiveness as a potential electronic material for emerging nanotechnology, artificially enhancing the spin-orbit coupling strength in graphene can open up new possibilities in graphene-based spintronics, and several theoretical and experimental works have addressed the effects of enhanced spin-orbit coupling in graphene by doping with heavy adatoms such as indium or thallium $\underline{\underline{7}}$, doping with $3 \mathrm{~d} / 5 \mathrm{~d}$ transition metal atoms $\mathrm{s}^{-10}$, and interfacing with metal substrates, e.g., $\mathrm{Ni}(111) \underline{11}$.

Due to band gap opening from broken out-of-plane inversion symmetry, gated bilayer graphene is a quantum valley-Hall insulator (QVHI) characterized by a quantized valley Chern number. In our recent work,$\frac{12}{\underline{1}}$ we have reported that the presence of Rashba spin-orbit coupling turns the gated bilayer graphene system from a QVHI into a $Z_{2}$ TI, with the phase boundary given by $\lambda_{R}^{2}=U^{2}+t_{\perp}^{2}$ where $\lambda_{R}, U$ and $t_{\perp}$ denote the strengths of Rashba spin-orbit coupling, interlayer potential difference and interlayer tunneling amplitude respectively. In this paper, we obtain low-energy effective Hamiltonians for the topological insulator phase, valid for small $U \ll$ $\lambda_{R}$ and below the topological phase transition, as well as for the quantum valley Hall phase above the phase transition. In the presence of different Rashba spin-orbit cou- pling strengths on the top and bottom layers $\left(\lambda_{R}^{1} \neq \lambda_{R}^{2}\right)$, we show that the topological insulator phase remains robust as long as $\lambda_{R}^{1} \lambda_{R}^{2}>t_{\perp}^{2}$. When the time-reversal symmetry is broken by an additional exchange field $M$, the bilayer system hosts different topological phases characterized by different Chern numbers $\mathcal{C}=2,4 \operatorname{sgn}(M)$ and valley Chern numbers $\mathcal{C}_{v}=2,4 \operatorname{sgn}(U)$, and the phase boundaries associated with the topological phase transitions are given by $U= \pm M$ and $U^{2}+t_{\perp}^{2}-M^{2}-\lambda_{R}^{2}=0$.

The rest of this paper is organized as follows. Section [II introduces the tight-binding and low-energy effective Hamiltonian of $A B$-stacked bilayer graphene in the presence of Rashba spin-orbit coupling, exchange field, and interlayer potential difference. In Section III we obtain the low-energy effective Hamiltonians of the $Z_{2}$ TI and the quantum valley Hall phases. We consider in Section IV the case of $A A$-stacked bilayer graphene. The effect of different Rashba spin-orbit coupling strengths on the top and bottom layers of $A B$-stacked bilayer graphene is considered in Section V. Finally, in Section VI we obtain the phase diagram of $A B$-stacked bilayer graphene as a function of $\lambda_{R}, U$ and $M$.

\section{SYSTEM HAMILTONIAN}

The tight-binding Hamiltonian of $A B$-stacked bilayer graphene in the presence of Rashba spin-orbit coupling, exchange field, and interlayer potential difference is given by 12,13

$$
\begin{aligned}
H_{\mathrm{BLG}}= & H_{\mathrm{SLG}}^{T}+H_{\mathrm{SLG}}^{B}+t_{\perp} \sum_{i \in T, j \in B} c_{i}^{\dagger} c_{j} \\
& +U \sum_{i \in T} c_{i}^{\dagger} c_{i}-U \sum_{i \in B} c_{i}^{\dagger} c_{i},
\end{aligned}
$$


where the first two terms $H_{\mathrm{SLG}}^{T, B}$ denote the monolayer graphene Hamiltonian for the top (T) and bottom (B) layer (see below), the third term represents the tunneling Hamiltonian that couples the top and bottom layers, and the last two terms take into account a potential difference $2 U$ between the top and bottom layers. The single-layer graphene Hamiltonian is: $2,9,14,15$

$$
H_{\mathrm{SLG}}=H_{0}+H_{R}+H_{M},
$$

with

$$
\begin{aligned}
& H_{0}=-t \sum_{\langle i j\rangle} c_{i}^{\dagger} c_{j} ; \\
& H_{R}=i t_{R} \sum_{\langle i j\rangle ; \alpha, \beta} \hat{\mathbf{e}}_{z} \cdot\left(s_{\alpha \beta} \times \mathbf{d}_{i j}\right) c_{i \alpha}^{\dagger} c_{j \beta} ; \\
& H_{M}=M \sum_{i ; \alpha, \beta} c_{i \alpha}^{\dagger} s_{\alpha \beta}^{z} c_{i \beta},
\end{aligned}
$$

where $\langle\ldots\rangle$ runs over all the nearest neighbor sites with hopping amplitude $t=2.6 \mathrm{eV}, \boldsymbol{s}$ are spin Pauli matrices with $\alpha$ and $\beta$ denoting up spin or down spin, and $c_{i \alpha}^{\dagger}$ $\left(c_{i \alpha}\right)$ is the electron creation (annihilation) operator on site $i . H_{R}$ describes the Rashba spin-orbit coupling with coupling strength $t_{R}$ and $\mathbf{d}_{i j}$ is a lattice vector pointing from sites $j$ to $i$, and $H_{M}$ the exchange field contribution with magnetization $M$.

In the momentum space $\stackrel{17}{ }$, we perform an expansion of the momentum about the valley points $K$ and $K^{\prime}$ and obtain the following eight-band effective Hamiltonian: ${ }^{12,13}$

$$
\begin{aligned}
H & =v\left(\eta \sigma_{x} k_{x}+\sigma_{y} k_{y}\right) \mathbf{1}_{s} \mathbf{1}_{\tau}+\frac{t_{\perp}}{2}\left(\sigma_{x} \tau_{x}-\sigma_{y} \tau_{y}\right) \mathbf{1}_{s} \\
& +\frac{\lambda_{R}}{2}\left(\eta \sigma_{x} s_{y}-\sigma_{y} s_{x}\right) \mathbf{1}_{\tau}+M s_{z} \mathbf{1}_{\sigma} \mathbf{1}_{\tau}+U \tau_{z} \mathbf{1}_{s} \mathbf{1}_{\sigma}
\end{aligned}
$$

where $\eta= \pm 1$ label the valley degrees of freedom, $\boldsymbol{\sigma}$ and $\tau$ are Pauli matrices representing the $A B$ sublattice and top-bottom layer degrees of freedom, $\mathbf{1}$ is a $2 \times 2$ identity matrix. The bare graphene Fermi velocity is given by $v=3 a t / 2$ with $a$ the lattice constant and Rashba spinorbit coupling is given by $\lambda_{R}=3 t_{R}$. For simplicity, we set the lattice constant $a$ to be unity henceforth.

\section{FOUR-BAND EFFECTIVE HAMILTONIAN}

In Ref. [12], we reported numerical tight-binding calculations showing that $A B$-stacked bilayer graphene under external interlayer potential undergoes a topological phase transition from a QVHI to a two-dimensional $Z_{2}$ TI. Figure1 1shows that, at the phase transition point, the bulk band gap of the bilayer graphene system obtained from Eq. (3) closes at the valley points at the zero energy (for comparison Figure 1 also shows the band structure of a pristine single-layer graphene). It is therefore possible to obtain low-energy Hamiltonian descriptions near band gap closing, which occurs when bilayer graphene turns into a TI from a semi-metal for small $U$ and at the

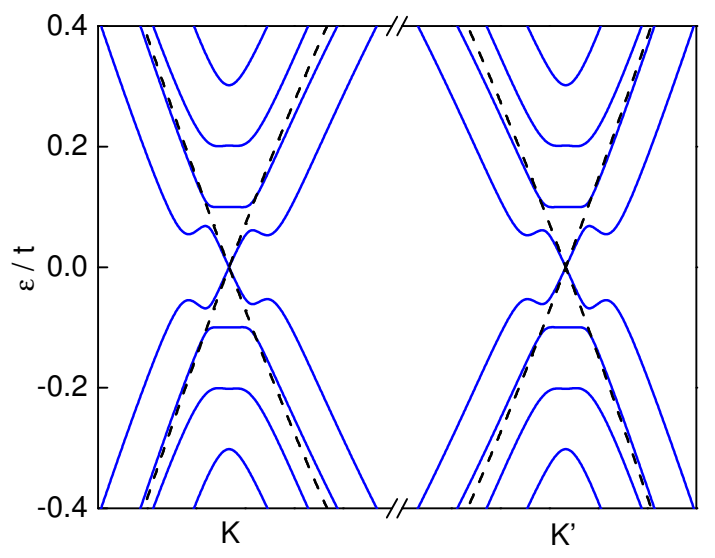

FIG. 1. (Color online) Solid line: bulk band structure of bilayer graphene at the phase transition point between the quantum valley-Hall and the $Z_{2}$ topological insulator phases. The parameters used are $U=0.10 t, t_{\perp}=0.143 t$ and $t_{R}=$ 0.058t. Dashed line: bulk band structure of pristine single layer graphene. The band gap closing at phase transition occurs precisely at the valleys $K$ and $K^{\prime}$.

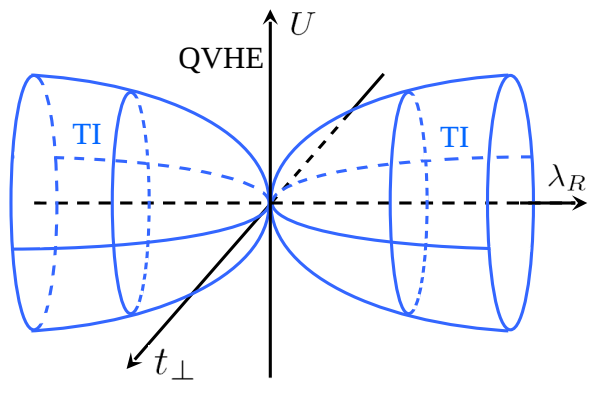

FIG. 2. (Color online) Phase diagram of bilayer graphene as a function of $\mathrm{U}, \lambda_{R}, t_{\perp}$. The region inside the ellipsoid corresponds to the $Z_{2}$ topological insulator state and outside region to the quantum valley Hall insulator phase. The phase boundary is given by Eq. (4)

phase transition point $U=U_{0}$ between TI and QVHI. In the following, we expand the eight-band Hamiltonian Eq. (3) up to the leading order in momentum $\boldsymbol{k}$ around the valley points and obtain a reduced four-band effective Hamiltonian that captures the low-energy physics of the system near phase transitions.

We assume equal Rashba spin-orbit coupling strengths in both top and bottom layers in this Section and address the effects of unequal Rashba strengths in Section $\mathrm{D}$ Figure 3 illustrates our results for the energy bands obtained numerically from the eight-band Hamiltonian in Eq. (3). We observe that the fourth band and the fifth band become inverted when the Rashba strength increases beyond a critical value signaling a topological phase transition. Imposing $\boldsymbol{k}=0$ in Eq. (3) gives the condition for gap closing of the bulk bands as 12

$$
\lambda_{R}^{2}=U^{2}+t_{\perp}^{2}
$$

The $\left(\mathrm{U}, \lambda_{R}, t_{\perp}\right)$ phase space is therefore divided into 


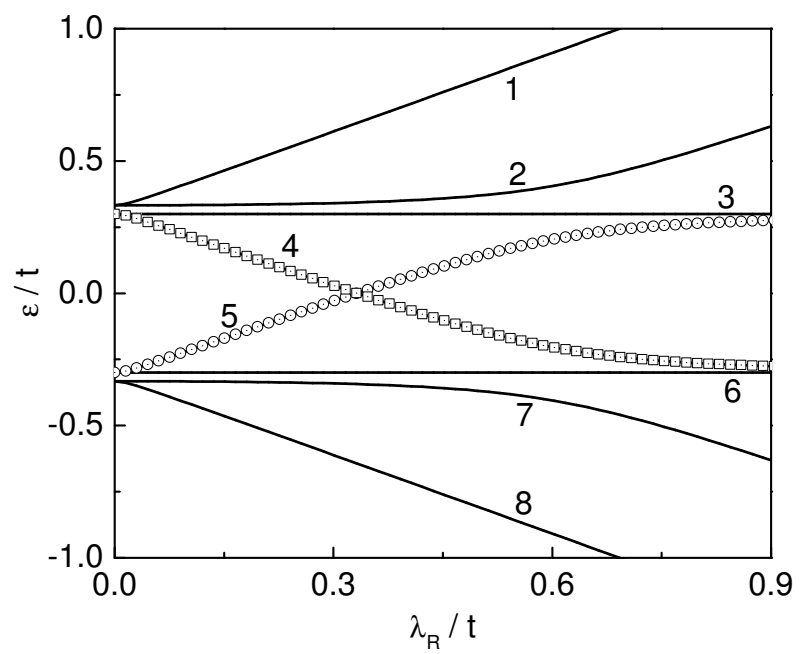

FIG. 3. Energy dispersion at valley $K(\mathbf{k}=0)$ as a function of Rashba spin-orbit coupling $\lambda_{\mathrm{R}}$ at fixed $\mathrm{U}=0.3 t$ and $t_{\perp}=$ $0.143 t$. One notices that band inversion occurs between band ' 4 ' and band ' 5 ' at the critical Rashba spin-orbit coupling value $\lambda_{R} \simeq 0.33 t$.

two regions as illustrated in Fig. 2, the system is in the $\mathcal{C}_{v}=4 \operatorname{sgn}(U)$ QVHI phase when $\lambda_{R}^{2}<U^{2}+t_{\perp}^{2}$, while it is in the $Z_{2}$ TI phase characterized by $\mathcal{C}_{v}=2 \operatorname{sgn}(U)$ and $Z_{2}=1$ when $\lambda_{R}^{2}>U^{2}+t_{\perp}^{2}$.

In Fig. 4, we plot the Chern number contributions from each valence band using the eight-band Hamiltonian in Eq. (3) near the valley $K$. Here, we fix the Rashba spinorbit coupling and interlayer coupling as $\lambda_{R} / t=0.617$ and $t_{\perp} / t=0.143$. The topological phase transition point occurs at $U_{0} \equiv \sqrt{\lambda_{R}^{2}-t_{\perp}^{2}}$. In the TI phase when $U<U_{0}$, the plot shows that the contribution to the total Chern number from each valence band varies as a function of $U$, and in particular there are two regimes where the Chern number contributions are distributed differently among the bands. For $U \rightarrow 0, C_{1,2,3,4}^{K}=-0.5,0.5,-1.5,0.5$ while for $U \rightarrow U_{0}^{-}, C_{1,2,3,4}^{K}=0.0,1.0,-2.0,0.0$. For the QVHI phase occurring when $U>U_{0}$, the Chern number contribution from each valence band is constant as a function of $U$ with $C_{1,2,3,4}^{K}=0.0,1.0,-2.0,-1.0$. In the following we study the low-energy physics of the TI and QVHI states in these three regimes.

\section{A. Near Semimetal-TI Phase Boundary: $U \rightarrow 0$ and $U \ll \lambda_{R}$}

In the basis $\left\{A_{1 \uparrow}, B_{1 \downarrow}, A_{2 \uparrow}, B_{2 \downarrow}, A_{1 \downarrow}, B_{1 \uparrow}, A_{2 \downarrow}, B_{2 \uparrow}\right\}$, the eight-band Hamiltonian Eq. (3) can be written at valley $K$ as:

$$
H_{\mathrm{K}}=\left[\begin{array}{cc}
H_{1} & T \\
T & H_{2}
\end{array}\right]
$$

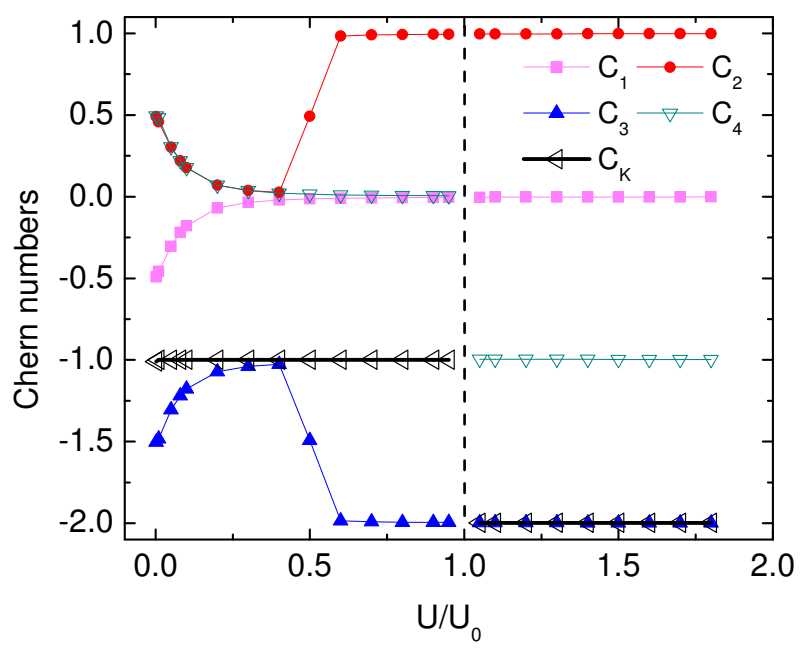

FIG. 4. (Color online) Chern number contribution from each valence band obtained from Eq. 3 at valley $K$ as a function of $U / U_{0}$. The Rashba spin-orbit coupling and interlayer tunneling are fixed. $U_{0}$ is the critical value (see the dashed line) of interlayer potential separating the 2D TI and QVH phases. For $U<U_{0}$, the total Chern number $\mathcal{C}_{K}=-1$, but the contribution from each valence band varies as a function of $U / U_{0}$. For $U>U_{0}$, the total Chern number $\mathcal{C}_{K}=-2$ and the contribution from each valence band is independent of $U / U_{0}$.

with

$$
\begin{gathered}
H_{1}=\left[\begin{array}{cccc}
+U & 0 & 0 & 0 \\
0 & +U & 0 & 0 \\
0 & 0 & -U & 0 \\
0 & 0 & 0 & -U
\end{array}\right], \\
H_{2}=\left[\begin{array}{cccc}
+U & i \lambda_{\mathrm{R}} & 0 & 0 \\
-i \lambda_{\mathrm{R}} & +U & 0 & 0 \\
0 & 0 & -U & i \lambda_{\mathrm{R}} \\
0 & 0 & -i \lambda_{\mathrm{R}} & -U
\end{array}\right],
\end{gathered}
$$

and

$$
T=\left[\begin{array}{cccc}
0 & v k_{-} & 0 & t_{\perp} \\
v k_{+} & 0 & 0 & 0 \\
0 & 0 & 0 & v k_{-} \\
t_{\perp} & 0 & v k_{+} & 0
\end{array}\right]
$$

In the limit $U \rightarrow 0$ and $U \ll \lambda_{R}, H_{1}$ and $H_{2}$ correspond respectively to the lower bands [i.e. $\varepsilon= \pm U$ ] and higher bands [i.e. $\varepsilon= \pm\left(\lambda_{\mathrm{R}} \pm U\right)$ ]. In the vicinity of $K$, the coupling $T$ between $H_{1}$ and $H_{2}$ becomes very weak. Therefore, the original eight-band Hamiltonian can be reduced to an effective four-band Hamiltonian: $\underline{18}$

$$
\begin{aligned}
H_{\mathrm{K}}^{\mathrm{eff}} & \simeq H_{1}-T H_{2}^{-1} T \\
& =\frac{1}{\lambda_{R}}\left[\begin{array}{cccc}
U \lambda_{R} & i v^{2} k_{-}^{2} & 0 & 2 i t_{\perp} v k_{-} \\
-i v^{2} k_{+}^{2} & U \lambda_{R} & 0 & 0 \\
0 & 0 & -U \lambda_{R} & i v^{2} k_{-}^{2} \\
-2 i t_{\perp} v k_{+} & 0 & -i v^{2} k_{+}^{2} & -U \lambda_{R}
\end{array}\right] .
\end{aligned}
$$




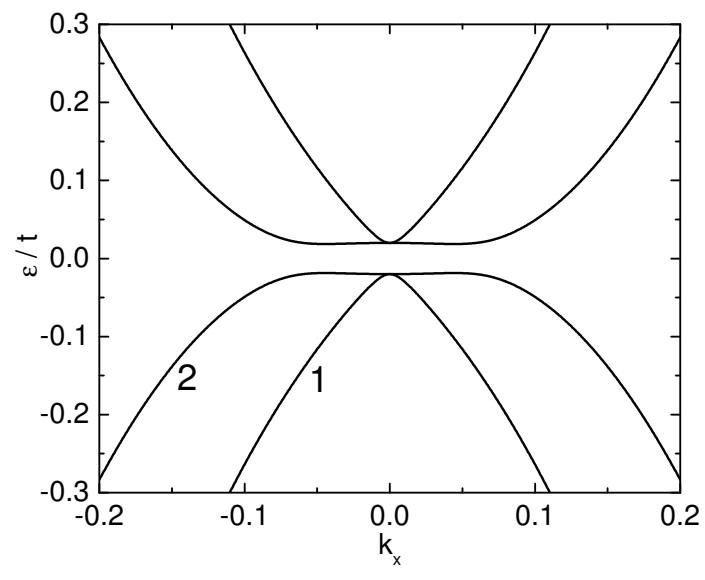

FIG. 5. Bulk band structure of the effective four-band model in the limit of $\lambda_{R} / U \gg 1$ along the profile of $k_{y}=0$. ' 1 ' and '2' label the two valence bands. Here, we choose $\lambda_{R} /=0.20 t$ and $U=0.02 t$. Note that the two conduction or valence bands touch at $k=0$.

Similarly, the eight-band Hamiltonian at valley $K^{\prime}$ in the basis of $\left\{A_{1 \downarrow}, B_{1 \uparrow}, A_{2 \downarrow}, B_{2 \uparrow}, A_{1 \uparrow}, B_{1 \downarrow}, A_{2 \uparrow}, B_{2 \downarrow}\right\}$ can be expressed as:

$$
H_{\mathrm{K}^{\prime}}=\left[\begin{array}{cc}
H_{1} & T_{0} \\
T_{0} & H_{2}
\end{array}\right]
$$

with

$$
T_{0}=\left[\begin{array}{cccc}
0 & -v k_{+} & 0 & t_{\perp} \\
-v k_{-} & 0 & 0 & 0 \\
0 & 0 & 0 & -v k_{+} \\
t_{\perp} & 0 & -v k_{-} & 0
\end{array}\right]
$$

Using the formula of Eq. (9), the resulting reduced four-band Hamiltonian can be written as:

$$
H_{\mathrm{K}^{\prime}}^{\mathrm{eff}} \simeq \frac{1}{\lambda_{R}}\left[\begin{array}{cccc}
U \lambda_{R} & i v^{2} k_{+}^{2} & 0 & -2 i t_{\perp} v k_{+} \\
-i v^{2} k_{-}^{2} & U \lambda_{R} & 0 & 0 \\
0 & 0 & -U \lambda_{R} & i v^{2} k_{+}^{2} \\
2 i t_{\perp} v k_{-} & 0 & -i v^{2} k_{-}^{2} & -U \lambda_{R}
\end{array}\right]
$$

Upon diagonalization of Hamiltonians in Eqs. (9) and (12), the energy dispersions at both $K$ and $K^{\prime}$ can be obtained and share the same form:

$$
\varepsilon= \pm \frac{\sqrt{\lambda_{R}^{2} U^{2}+\epsilon_{k}^{4}+2 \epsilon_{k}^{2} t_{\perp}^{2} \pm 2 \epsilon_{k}^{2} \sqrt{\lambda_{R}^{2} U^{2}+t_{\perp}^{4}+\epsilon_{k}^{2} t_{\perp}^{2}}}}{\lambda_{R}},
$$

where $\epsilon_{k}=v k$.

Figure 5 plots the bulk band structure of the four-band effective Hamiltonian in Eq. (13) along $k_{y}=0$. One can see that a bulk band gap opens and the resulting conduction and valence bands touch at $k_{x}=0$. The bulk band gap opening signals an insulating state. To reveal its topological property, we have evaluated the Berry phase contribution from the occupied valence bands below the band gap. In a continuum model Hamiltonian, the Chern number is calculated by integrating the Berry curvature in the entire momentum space:

$$
\mathcal{C}=\frac{1}{2 \pi} \sum_{n=1,2} \int_{-\infty}^{+\infty} \int_{-\infty}^{+\infty} d k_{x} d k_{y} \Omega_{n}\left(k_{x}, k_{y}\right)
$$

where $\Omega_{n}\left(k_{x}, k_{y}\right)$ is the momentum-space Berry curvature at $\left(k_{x}, k_{y}\right)$ of the $n$-th band, and is given by

$$
\Omega_{n}(\boldsymbol{k})=-\sum_{n^{\prime} \neq n} \frac{2 \operatorname{Im}\left\langle\psi_{n \boldsymbol{k}}\left|v_{x}\right| \psi_{n^{\prime} \boldsymbol{k}}\right\rangle\left\langle\psi_{n^{\prime} \boldsymbol{k}}\left|v_{y}\right| \psi_{n \boldsymbol{k}}\right\rangle}{\left(\omega_{n^{\prime}}-\omega_{n}\right)^{2}}
$$

where $\omega_{n} \equiv \varepsilon_{n} / \hbar$, and $v_{x(y)}$ is the velocity operator along the $x(y)$-direction.

In Fig. 6. we display the Berry curvature distribution $\Omega$ along $k_{y}=0$ for both valleys $K$ and $K^{\prime}$. One observes that the Berry curvatures are exactly opposite at the two valleys $K$ and $K^{\prime}$ for each band. In particular, we find that the total Berry curvatures around $K$ or $K^{\prime}$ do not share the same sign in the whole momentum space, in contrast with the Berry curvature distribution in the quantum anomalous Hall effect in single layer graphene ${ }^{9}$ or the conventional QVHI in graphene due to the presence of staggered $\mathrm{AB}$ sublattice potential. $\underline{10,19}$

By numerically evaluating the integration in the momentum space, the Chern numbers at valleys $\mathrm{K}$ and $\mathrm{K}$ ' are found to be

$$
\mathcal{C}_{K}=-\mathcal{C}_{K^{\prime}}=\operatorname{sgn}(U),
$$

in which the Chern number contribution from each valence band are

$$
\begin{aligned}
& \mathcal{C}_{K}^{1}=-\mathcal{C}_{K^{\prime}}^{1}=-\frac{1}{2} \operatorname{sgn}(U), \\
& \mathcal{C}_{K}^{2}=-\mathcal{C}_{K^{\prime}}^{2}=+\frac{3}{2} \operatorname{sgn}(U),
\end{aligned}
$$

where the supscripts label the valence band indices in Fig. [5.

From the principle of bulk-edge correspondence, one expect that there is only one pair of edge states propagating along the system boundaries. Since time-reversal symmetry is preserved in the our system, one concludes that this nontrivial insulating state belongs to the $Z_{2}$ TI class. Therefore, the edge modes are robust against weak non-magnetic impurities. Moreover, since different valleys are encoded into the counter-propagating edge channels,$\frac{12}{2}$ they are further protected by the large momentum separation as long as inter-valley scattering is forbidden. As a consequence, these edge modes are also robust against smooth non-magnetic and magnetic impurities. From Eq. (16), the valley Chern number of this $Z_{2}$ TI state is:

$$
\mathcal{C}_{v}=\mathcal{C}_{K}-\mathcal{C}_{K^{\prime}}=\frac{2 e^{2}}{h} \operatorname{sgn}(U),
$$

which is half of that in the conventional QVHI $\underline{13}$ 

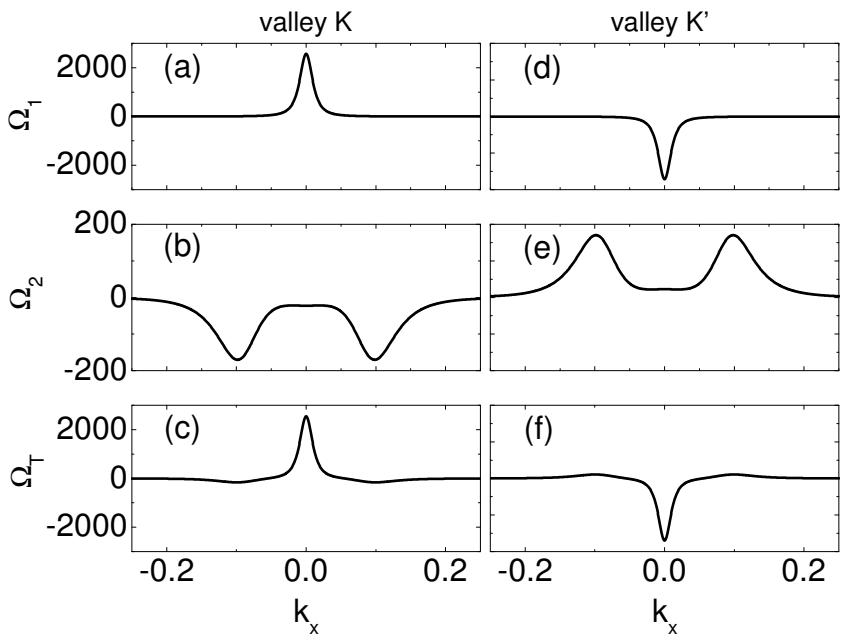

FIG. 6. Berry curvature distribution $\Omega$ at valleys $K$ and $K^{\prime}$ along $k_{y}=0$ of the effective four-band model in the limit of $\lambda_{R} / U \gg 1$. Upper panels: Berry curvature distribution $\Omega_{1}$ for the lowest valence band labeled as ' 1 ' in Fig. [5. Middle panels: Berry curvature distribution $\Omega_{2}$ for the valence band close to the band gap labeled as ' 2 ' in Fig. 5 Lower panels: the total valence band Berry curvature distribution $\Omega_{T}$. The parameters adopted here are the same as those used in Fig. 5

\section{B. Near TI-QVHI Phase Boundary: $U \rightarrow \sqrt{\lambda_{R}^{2}-t_{\perp}^{2}}$}

In the following discussion, we set $\lambda_{R}$ and $t_{\perp}$ as fixed, and allow $U$ to slightly deviate from the phase transition point $U_{0}$, i.e., $U=U_{0}+\Delta$, where $U_{0}=\sqrt{\lambda_{R}^{2}-t_{\perp}^{2}}, \Delta>$ 0 and $<0$ correspond respectively to the QVHI and TI phases.

When the topological phase transition occurs, the bulk band gap closes at the valley points $K / K^{\prime}$. Hence, at the critical value $U_{0}$ and $\boldsymbol{k}=0$, the system Hamiltonian in the basis of $\left\{B_{1 \downarrow}, A_{2 \uparrow}, A_{1 \uparrow}, A_{2 \downarrow}, B_{2 \uparrow}, B_{2 \downarrow}, B_{1 \uparrow}, A_{1 \downarrow}\right\}$ can be expressed as

$$
H=\left[\begin{array}{cccccccc}
U_{0} & 0 & 0 & 0 & 0 & 0 & 0 & 0 \\
0 & -U_{0} & 0 & 0 & 0 & 0 & 0 & 0 \\
0 & 0 & U_{0} & 0 & t_{\perp} & 0 & 0 & 0 \\
0 & 0 & 0 & -U_{0} & -i \lambda_{R} & 0 & 0 & 0 \\
0 & 0 & t_{\perp} & i \lambda_{R} & -U_{0} & 0 & 0 & 0 \\
0 & 0 & 0 & 0 & 0 & -U_{0} & 0 & t_{\perp} \\
0 & 0 & 0 & 0 & 0 & 0 & U_{0} & i \lambda_{R} \\
0 & 0 & 0 & 0 & 0 & t_{\perp} & -i \lambda_{R} & U_{0}
\end{array}\right] 20
$$

The eigenenergies can be obtained from the above as $\varepsilon=$ $0,0, \pm U_{0}, \pm \varepsilon_{1}, \pm \varepsilon_{2}$, where $\varepsilon_{1}=-U_{0} / 2+\sqrt{8 \lambda_{R}^{2}+U_{0}^{2}} / 2$ and $\varepsilon_{2}=-U_{0} / 2-\sqrt{8 \lambda_{R}^{2}+U_{0}^{2}} / 2$. The former four correspond to the low-energy part, while the latter four correspond to the high-energy part. Based on our analysis of the Berry curvature from the tight-binding model, the valley Chern number arises only from the low-energy bands and the high-energy bands give no contribution.

The unitary transformation matrix that diagonalizes Eq. (20) is presented in the Appendix. After some manipulations, the effective Hamiltonian can be obtained from

$$
H_{\text {eff }}=H_{P}-T H_{Q}^{-1} T^{\dagger},
$$

where explicit expressions of $H_{P}, T, H_{Q}$ are also given in the Appendix. The effective Hamiltonian to first order in $\boldsymbol{k}$ is then given by

$$
H_{\mathrm{eff}}^{(1)}=\left[\begin{array}{cccc}
-\frac{U_{0}^{2}}{\lambda_{R}^{2}} \Delta & -i \frac{t_{\perp}}{\lambda_{R}} k_{-} & 0 & -\frac{U_{0}}{\sqrt{2} \lambda_{R}} k_{+} \\
i \frac{t_{\perp}}{\lambda_{R}} k_{+} & \frac{U_{0}^{2}}{\lambda_{R}^{2}} \Delta & \frac{U_{0}}{\sqrt{2} \lambda_{R}} k_{-} & 0 \\
0 & \frac{U_{0}}{\sqrt{2} \lambda_{R}} k_{+} & \Delta+U_{0} & 0 \\
-\frac{U_{0}}{\sqrt{2} \lambda_{R}} k_{-} & 0 & 0 & -\Delta-U_{0}
\end{array}\right],
$$

In the following, we show that the first-order effective Hamiltonian at valley $K$ is sufficient to capture the topological phase transition between the QVHI and TI phases. Figure 7 displays the band structures along $k_{y}=$ 0 for three different values of $\Delta / t=-0.03,0.00,0.03$ at fixed $U_{0} / t=0.30$ and $\lambda_{R} / t=0.33$. One observes that when $\Delta$ increases from negative to positive, closing and reopening of the bulk band gap occur as expected. Figure 8 plots the Berry curvatures along $k_{y}=0$ of the two valence bands below the band gap and the total Berry curvature for $\Delta / t= \pm 0.03$. The Berry curvatures for the first valence band $\Omega_{1}$ in both cases are similar sharing the same sign [panels (a) and (d)]. For the second valence band, the Berry curvatures for $\Delta / t=-0.03$ exhibit both positive and negative signs [panel (b)], but for $\Delta=+0.03$, they are both negative [panel (e)]. As a consequence, the total berry curvatures at $\Delta / t=-0.03$ include both positive and negative contributions [panel (c)], and those at $\Delta=+0.03$ share the same negative sign. By using Eq. (14), the Chern numbers acquired by the valence bands at valley $K$ is evaluated as $\mathcal{C}_{K}=-1,-2$ for $\Delta=-0.03,+0.03$ respectively. Following a similar derivation, one obtains the effective Hamiltonian at valley $K^{\prime}$ with the corresponding Chern numbers $\mathcal{C}_{K^{\prime}}=1,2$ for $\Delta=-0.03,+0.03$ respectively. In this way, the valley Chern number in the topological insulator phase is $\mathcal{C}_{v}=\mathcal{C}_{K}-\mathcal{C}_{K^{\prime}}=-2$ while that in the quantum valley Hall phase is $\mathcal{C}_{v}=\mathcal{C}_{K}-\mathcal{C}_{K^{\prime}}=-4$.

In all the three limits, the valley-Chern numbers from the resulting four-band effective Hamiltonians are consistent with those from the direct eight-band full Hamiltonian.

\section{AA-STACKED BILAYER GRAPHENE}

Bilayer graphene is composed of two monolayers of graphene, usually arranged in $A B$ or $A A$ stacking pattern. In previous Sections, we have predicted a $Z_{2}$ TI phase in the $A B$ stacking configuration. It therefore becomes a natural question to ask whether the $A A$ stacking configuration can also host a $Z_{2}$ TI phase. In this Section, we demonstrate that the $A A$-stacked bilayer graphene does not realize a $Z_{2}$ topological insulator state in the presence of Rashba spin-orbit coupling and interlayer potential. 


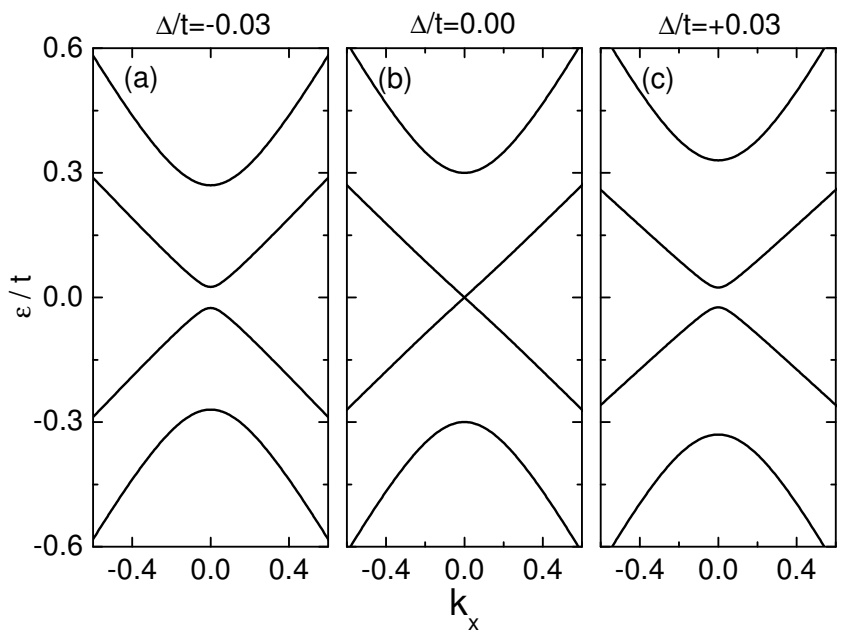

FIG. 7. Band structure obtained from the effective Hamiltonian Eq. (22) along $k_{y}=0$. The parameters for the phase transition point are set to be $U_{0} / t=0.30$ and $\lambda / t=0.33$. (a) $\Delta / t=-0.03$; (b) $\Delta / t=0.00$; (c) $\Delta / t=+0.03$. The bulk band gap decreases toward zero when $\Delta / t=0.00$ and then reopens when $\Delta$ becomes positive.
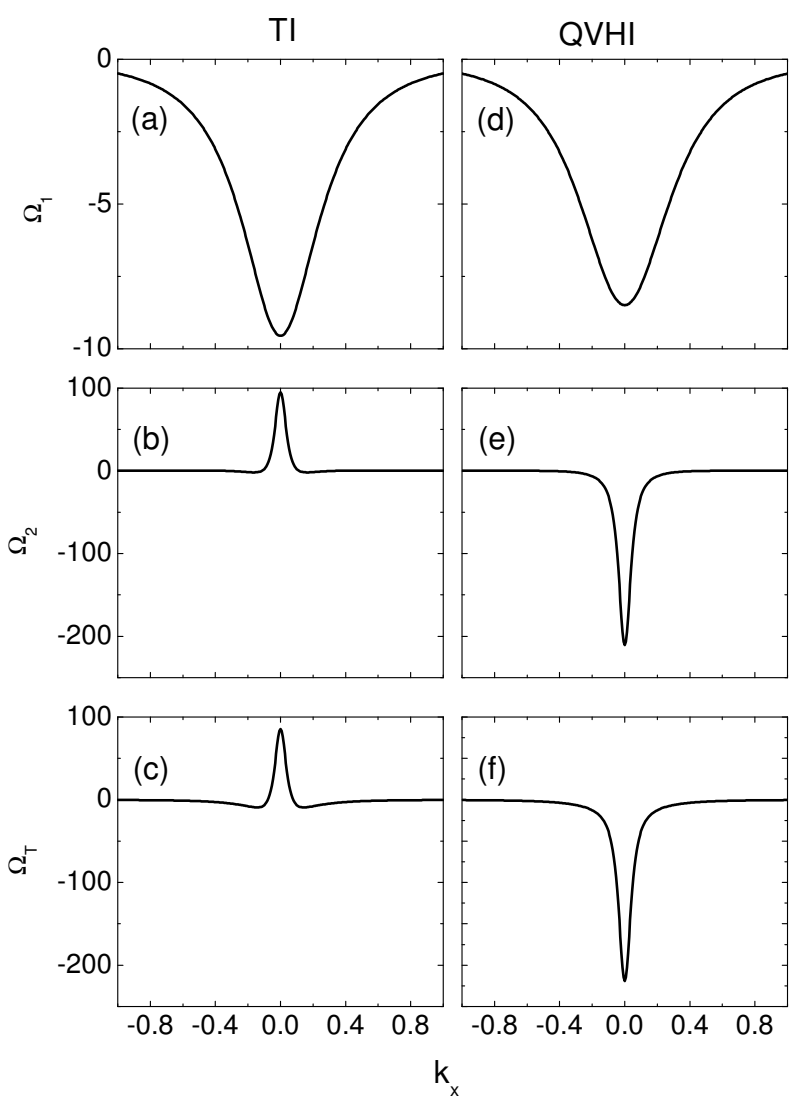

FIG. 8. Berry curvature distribution $\Omega$ along $k_{y}=0$ for $\Delta=$ \pm 0.03 . Other parameters are $U_{0} / t=0.30$ and $\lambda / t=0.33$. (a)-(c): Berry curvature distribution for each valence bands and the summary of all valence bands at $\Delta / t=-0.03$. (d)-(f) Berry curvature distribution for each valence bands and the summary of all valence bands at $\Delta / t=+0.03$.
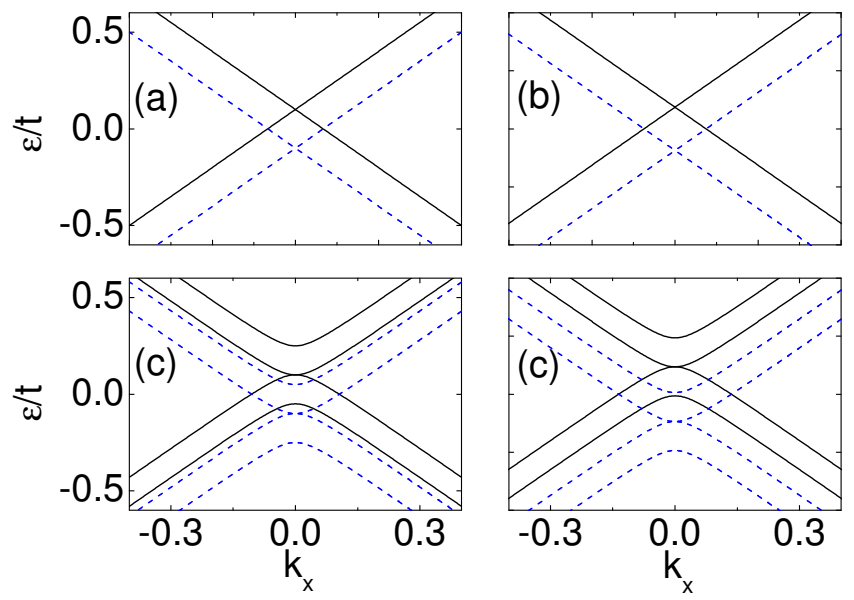

FIG. 9. (Color online) Bulk band structures of $A A$-stacked bilayer graphene along $k_{y}=0$ at valley $K$. (a) Pristine graphene, $U / t=0$ and $\lambda_{R} / t=0$; (b) $U=0.10 t$ and $\lambda_{R}=0$; (c) $U=0$ and $\lambda_{R}=0.15 t$; (d) $U=0.10 t$ and $\lambda_{R}=0.15 t$. Solid and dashed bands correspond to the top and bottom layers, respectively. Here the interlayer coupling is set to be $t_{\perp}=0.10 t$.

Figure 9 plots the bulk band structures of $A A$-stacked bilayer graphene along $k_{y}=0$ obtained from the lowenergy continuum model Hamiltonian at valley $K \stackrel{20}{\underline{20}}$ For pristine AA-stacked graphene, the linear Dirac dispersion near valley $K$ still holds as shown in panel (a), resembling two copies of monolayer graphene with a relative shift of $2 t_{\perp}$ (solid and dashed lines are used to label the bands from top and bottom layers). In the presence of an interlayer potential difference, there is no bulk band gap opening [see panel (b)] since the inversion symmetry with respect to the graphene plane is not violated. If only the Rashba spin-orbit coupling is turned on, one finds that again the resulting band structure is a combination of two copies of the monolayer graphene's band structures with a relative shift [see panel (c)]. When both Rashba spin-orbit coupling and interlayer potential difference are present, no bulk band gap appears. We therefore conclude that inversion symmetry breaking is a necessary requirement for the $Z_{2}$ TI in the bilayer graphene system. In addition to $A A$ and $B B$ stacking, twisted bilayer graphene presents another possibility which has attracted much recent interest. $\stackrel{21-25}{2}$ In future works, it will be interesting to study the possibility of inducing a $Z_{2}$ TI in a twisted bilayer graphene.

\section{EFFECTS OF DIFFERENT RASHBA SPIN-ORBIT COUPLINGS ON TWO LAYERS}

We have studied the $Z_{2}$ TI state while assuming the same Rashba spin-orbit coupling in the top and bottom layers of the $A B$-stacked bilayer graphene. In bilayer graphene, Rashba spin-orbit coupling is extremely weak, and one has to employ external means to enhance the 
Rashba spin-orbit coupling, e.g., doping it with heavy metal atoms or interfacing it with substrates. Therefore, the resulting Rashba spin-orbit couplings are likely to be different on the top and bottom layers. In the following, we discuss the effect of different top and bottom Rashba spin-orbit coupling strengths on the resulting TI state.

We adopt the low-energy continuum Hamiltonian Eq. (3) and assume different values of top and bottom Rashba spin-orbit couplings $\lambda_{1} \neq \lambda_{2}$. We first consider the case when both $\left|\lambda_{1}-\lambda_{2}\right|$ and $U$ are small. In this case, we found from our numerical calculations that although the conduction and valance bands are no longer symmetric with respect to $\varepsilon=0$, the bands still close exactly at the valley points $K$ and $K^{\prime}$; it is thus possible to obtain an analytic formula that describes the band closing condition. After imposing $\boldsymbol{k}=0$, one finds that two of the eigenenergies are $\varepsilon= \pm U$, while the remaining six ones satisfy the following equations:

$$
\varepsilon^{3}+(-1)^{i} U \varepsilon^{2}-\left(\lambda_{i}^{2}+U^{2}+t_{\perp}^{2}\right) \varepsilon-(-1)^{i} U\left(U^{2}-\lambda_{i}^{2}+t_{\perp}^{2}\right)=0, i=1,2 .
$$

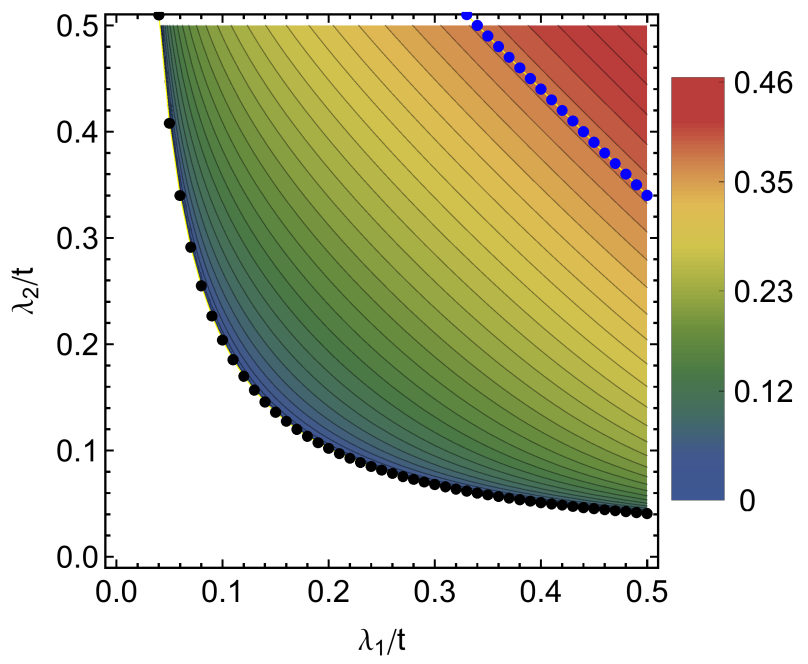

FIG. 10. (Color online) The interlayer potential $U$ that closes the band gap at the valley $\mathrm{K}$ point as a function of Rashba spin-orbit couplings $\lambda_{1}$ and $\lambda_{2}$, as predicted by Eq. (26). Colors represent the amplitude of $U$. It can be seen that while such contours are hyperbolas for small $U$ (see the dotted line in the lower part of the graph), they become linear for larger $U$ (see the straight lines in the upper-right corner). In addition, the constraint of $\lambda_{1} \lambda_{2}>t_{\perp}^{2}$ is clearly demonstrated in this plot.

We search for the condition when the top of the valence band and the bottom of the conduction band touch, closing the bulk band gap at the $K$ or $K^{\prime}$ point. This can be translated into the condition that the two equations in Eq. (22) have a common real-valued solution that lies between $-U$ and $U$. The latter condition is necessary in order to rule out the scenario that two higher (lower) bands touch at the $K$ point.

It turns out that the numerical search for a common solution is not as simple as the case with identical Rashba effects in Ref. [12], where we can directly require the common solution to be $\varepsilon=0$. In the present case, however, the band closing point is no longer fixed at $\varepsilon=0$, which makes it difficult to obtain a simple analytical solution. Instead, we opt to solve for $\lambda_{i}$ (and not for $\varepsilon$ ) from Eq. (22), obtaining

$$
\begin{aligned}
& \lambda_{1}^{2}=\frac{U-\varepsilon_{0}}{U+\varepsilon_{0}}\left(U^{2}+t_{\perp}^{2}-\varepsilon_{0}^{2}\right), \\
& \lambda_{2}^{2}=\frac{U+\varepsilon_{0}}{U-\varepsilon_{0}}\left(U^{2}+t_{\perp}^{2}-\varepsilon_{0}^{2}\right),
\end{aligned}
$$

where $\varepsilon_{0}$ is the common solution of the two equations in Eq. (22). Then the band-closing point can be analytically obtained by dividing Eq. (23) by Eq. (24):

$$
\varepsilon_{0}=\frac{\lambda_{2}-\lambda_{1}}{\lambda_{2}+\lambda_{1}} U
$$

In order to have a band gap closing, these parameters must also satisfy the following condition

$$
t_{\perp}^{2}+\frac{4 \lambda_{1} \lambda_{2}}{\left(\lambda_{1}+\lambda_{2}\right)^{2}} U^{2}=\lambda_{1} \lambda_{2},
$$

which is derived by multiplying Eqs. (23) and (24). It is reassuring to see that when $\lambda_{1}=\lambda_{2}$, this condition does reduce to the one given in Eq. (44). One can also rewrite Eq. (26) by expressing the interlayer potential difference $U$ as a function of $\lambda_{1}$ and $\lambda_{2}$ :

$$
U= \pm \frac{\lambda_{1}+\lambda_{2}}{2} \sqrt{1-\frac{t_{\perp}^{2}}{\lambda_{1} \lambda_{2}}}
$$

where we see that the band gap at the $K$ or $K^{\prime}$ point will not be able to close if $\lambda_{1} \lambda_{2}<t_{\perp}^{2}$.

Figure 10 plots the interlayer potential difference $U$ in the $\left(\lambda_{1}, \lambda_{2}\right)$ plane that satisfies the band gap closing condition Eq. (26). Colors represent the strength of $U$. In the blank region, no band gap closing occurs under the constraint of $\lambda_{1} \lambda_{2}>t_{\perp}^{2}$. In the limit of small potential difference $U$, the contours of $U$ behave as hyperbolas given by $\lambda_{1} \lambda_{2}=t_{\perp}^{2}$ (see the black dotted line), while in the large $U$ limit, the contours tend to straight lines given by $\lambda_{1}+\lambda_{2}=2 U$.

To verify the correctness of the analytical expression of the band gap closing in Eq. (26), we compare it with band gap results from direct numerical diagnolization of the eight-band continuum model Hamiltonian at valley 


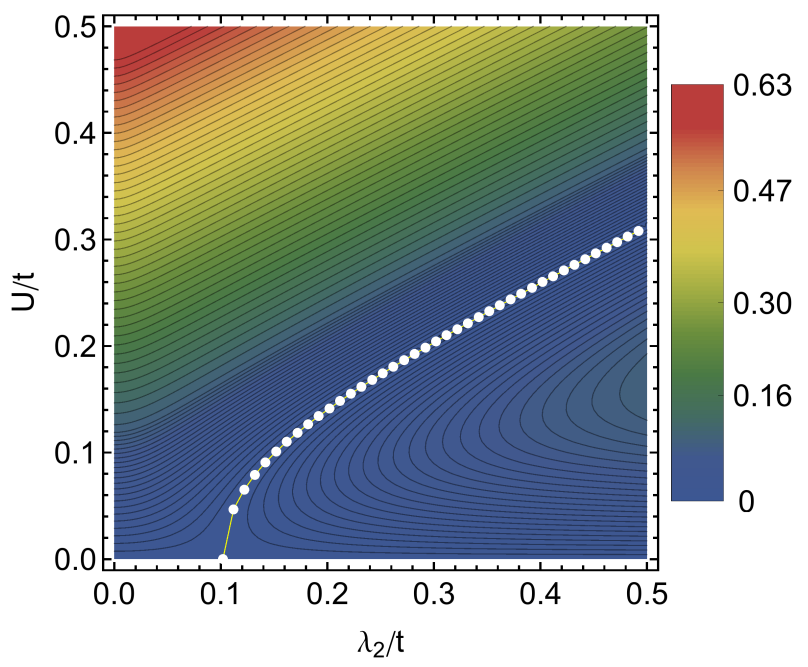

FIG. 11. (Color online) Numerically obtained band gap at valley $K$ point as a function of the interlayer potential $U$ and bottom-layer Rashba spin-orbit coupling $\lambda_{2}$ at a fixed toplayer Rashba spin-orbit coupling $\lambda_{1} / t=0.2$. Color measures of the size of the band gap. The white dots are plotted from Eq. (26). The analytical condition Eq. (26) for band gap closing overlaps with the numerical result.

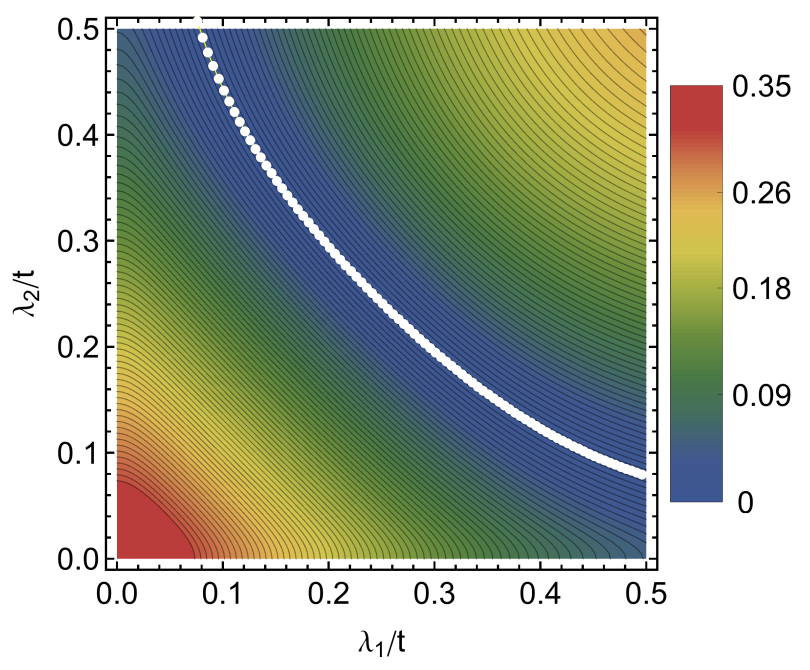

FIG. 12. (Color online) Numerically computed band gap at valley $K$ point as a function of Rashba spin-orbit couplings in both top and bottom layers $\lambda_{1}$ and $\lambda_{2}$. In this plot the interlayer potential difference is set as $U=0.20 t$. Color measures the size of the band gap in units of $t$. The white dots are plotted from Eq. (26). The analytical result for the band gap closing condition agrees well with that obtained from numerical calculations.

$K / K^{\prime}$. In Fig. 11, we plot the band gap at $K$ point as a function of interlayer potential difference $U$ and Rashba spin-orbit coupling $\lambda_{2}$ at a fixed $\lambda_{1}=0.20 t$. White dots plot the analytic result Eq. (26) corresponding to the boundary for band gap closing. We find that it agrees well with the numerically obtained condition for band gap closing. Similarly, in Fig. 12, we plot the band gap at $K$ point as a function of the two different Rashba spin-orbit couplings $\lambda_{1}$ and $\lambda_{2}$ at a fixed interlayer potential difference $U=0.2 t$. The white dots obtained from Eq. (26) fit exactly where the band gap closes at the valley $K / K^{\prime}$ point. Therefore, the analytical phase boundary in Eq. (26) indeed captures the band gap closing condition at valley $K / K^{\prime}$.

For very different $\lambda_{1}$ and $\lambda_{2}$ and a large $U$, we find that the conduction and valence bands can close indirectly at different momenta, and as a result there is no global bulk gap even though the direct gap at the valley points is nonzero. The global bulk gap is the smallest energy difference between the conduction band and the valence band across the entire Brillouin zone.

In Fig. 13, we show the comparison between the numerically computed band gap (circle or triangle) and direct band gap at the valley points given by Eq. (26) (solid line). One observes that for large differences in $\lambda_{1}$ and $\lambda_{2}$ and for large $U$, the numerically computed gap deviates from Eq. (26), indicating that band gaps close indirectly at different momenta.

To examine the nontrivial topology of the phases before and after the band gap closing, we calculate the valley-Chern numbers using the continuum model and the $Z_{2}$ topological number using the tight-binding model presented in Ref. [26]. As depicted in Fig. 13] before the phase transition, the system hosts a QVHI phase with $\mathcal{C}_{v}=\mathcal{C}_{K}-\mathcal{C}_{K^{\prime}}=4$, while after the phase transition, it enters into a TI phase with $Z_{2}=1$. Note that the TI state is simultaneously a QVHI state characterized by $\mathcal{C}_{v}=2$. These results are consistent with our findings in the presence of identical Rashba spin-orbit couplings $\underline{12}$ Therefore, we have shown that the $Z_{2}$ TI state we predicted in Ref. 12] remains robust when the top and bottom layer Rashba spin-orbit coupling strengths become different.

\section{EXCHANGE FIELD EFFECT}

This section is dedicated to investigate the exchange field effect on the QVHI state and $Z_{2}$ TI state in gated bilayer graphene. In Ref. [9], we have found that a bulk band gap will open in monolayer graphene in the presence of both Rashba spin-orbit coupling and exchange field, inducing a quantum anomalous-Hall phase $\mathrm{e}^{27-33}$. In Ref. [13], we have shown that in gated bilayer graphene, when the exchange field $M$ is larger than the interlayer potential difference $U$, i.e. $M>U$, the system undergoes a topological phase transition from the $\mathcal{C}_{v}=4$ QVHI phase into a $\mathcal{C}=4$ quantum anomalous-Hall phase. In the following, we supplement this result with a new phase boundary that has been overlooked in Ref. [13]. We also discuss how the QVHI state with $\mathcal{C}_{v}=1,2$ evolves in the presence of the exchange field.

For simplicity, we set the Rashba spin-orbit couplings to be the same in both layers in this discussion. We start from the low energy continuum model Eq. ((프) ), and con- 


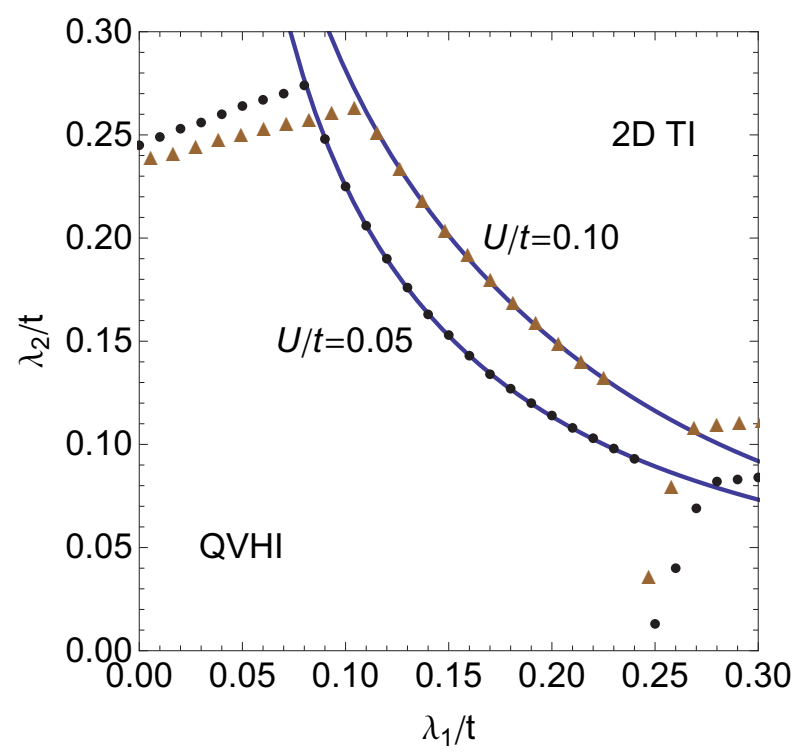

FIG. 13. (Color online) Comparison between the conditions for global bulk gap closing obtained numerically (circles and triangles) and local bulk gap closing predicted from Eq. (26) (solid line), for interlayer potential difference $U=0.05 t$ and $U=0.10 t$.

sider the following ingredients in our system: interlayer potential difference $U$, Rashba SOC in both layers $\lambda_{R}$, and exchange field $M$. The conduction and valence bands are symmetric about $\varepsilon=0$, and the bulk band gap closes at exactly the valley $K / K^{\prime}$ point. The energy dispersion of the eight-band Hamiltonian at valley $K$ is determined by the following equations:

$$
\begin{aligned}
& \varepsilon= \pm(M-U) \\
& \lambda_{R}^{2}(M \pm \varepsilon+U)=(M \pm \varepsilon+U)\left[U^{2}+t_{\perp}^{2}-(M-\varepsilon)^{2}\right] .
\end{aligned}
$$

The equations for valley $K^{\prime}$ can be obtained from the above by replacing $U \rightarrow-U$. By imposing $\varepsilon=0$, we obtain the following bulk gap closing condition

$$
M^{2}-U^{2}=0,
$$

which has been reported in Ref. [13]. In addition, if $\lambda_{R} \neq$ $\pm t_{\perp}$ and $U^{2}+t_{\perp}^{2}-\lambda_{R}^{2} \geq 0$ then a second gap closing condition

$$
U^{2}+t_{\perp}^{2}-M^{2}-\lambda_{R}^{2}=0,
$$

is also possible. These two conditions signify two topological phase transitions and give the corresponding phase transition boundaries.

In the above discussions, we have omitted a very important case in the presence of vanishing Rashba SOC $\lambda_{R}=0$. It is known that a large exchange field will close the bulk band gap induced from potential difference and results in a metallic phase. Most importantly, the band gap closing is not always exactly at the valley points as in other phase transitions we have discussed. After rearranging the Hamiltonian, the $8 \times 8$ Hamiltonian can be

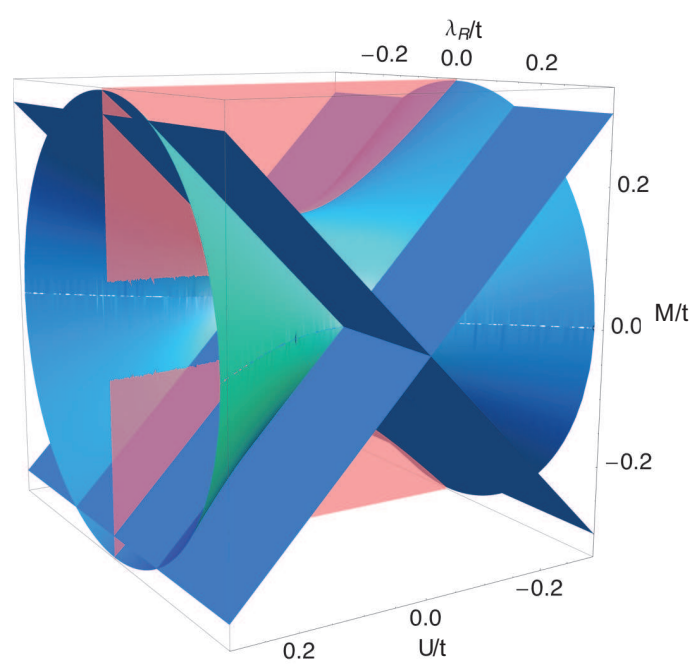

FIG. 14. (Color online) Phase diagram in the $\left(M, \lambda_{R}, U\right)$ space. The whole phase space is divided into various topological phases. Here, we only show the phase boundaries. (1) The mutual vertical planes determined by Eq. (28) separate the quantum valley Hall and quantum anomalous Hall phases. The uniparted hyperboloid determined by Eq. (29) is used to separate different sub-phases. Note: in the plane of $\lambda_{R}=0$, the red color represents the metallic phase.

written as:

$$
H_{K}=\left[\begin{array}{cc}
H_{1} & T \\
T & H_{2}
\end{array}\right]
$$

where

$$
\begin{aligned}
& H_{1}=\left[\begin{array}{cccc}
U+M & 0 & 0 & 0 \\
0 & U-M & 0 & 0 \\
0 & 0 & -U+M & 0 \\
0 & 0 & 0 & -U-M
\end{array}\right], \\
& H_{2}=\left[\begin{array}{cccc}
U-M & 0 & 0 & 0 \\
0 & U+M & 0 & 0 \\
0 & 0 & -U-M & 0 \\
0 & 0 & 0 & -U+M
\end{array}\right],
\end{aligned}
$$

and

$$
T=\left[\begin{array}{cccc}
0 & v k_{-} & 0 & t_{\perp} \\
v k_{+} & 0 & 0 & 0 \\
0 & 0 & 0 & v k_{-} \\
t_{\perp} & 0 & v k_{+} & 0
\end{array}\right]
$$

Due to the particle-hole symmetry, the bulk gap closing must occur at $\varepsilon=0$. Therefore, the equation should satisfy the following

$$
\begin{aligned}
& U^{2} t_{\perp}^{2}+M^{2}+\left(U^{2}-v^{2} k^{2}\right)^{2}-M^{2}\left[t_{\perp}^{2}+2\left(U^{2}+v^{2} k^{2}\right)\right] \\
& =0 .
\end{aligned}
$$

In order to have a real solution, we need

$$
\left(M^{2}+U^{2}\right)^{2}-\left(M^{2}-U^{2}\right)\left(M^{2}-U^{2}-t_{\perp}^{2}\right) \geq 0,
$$



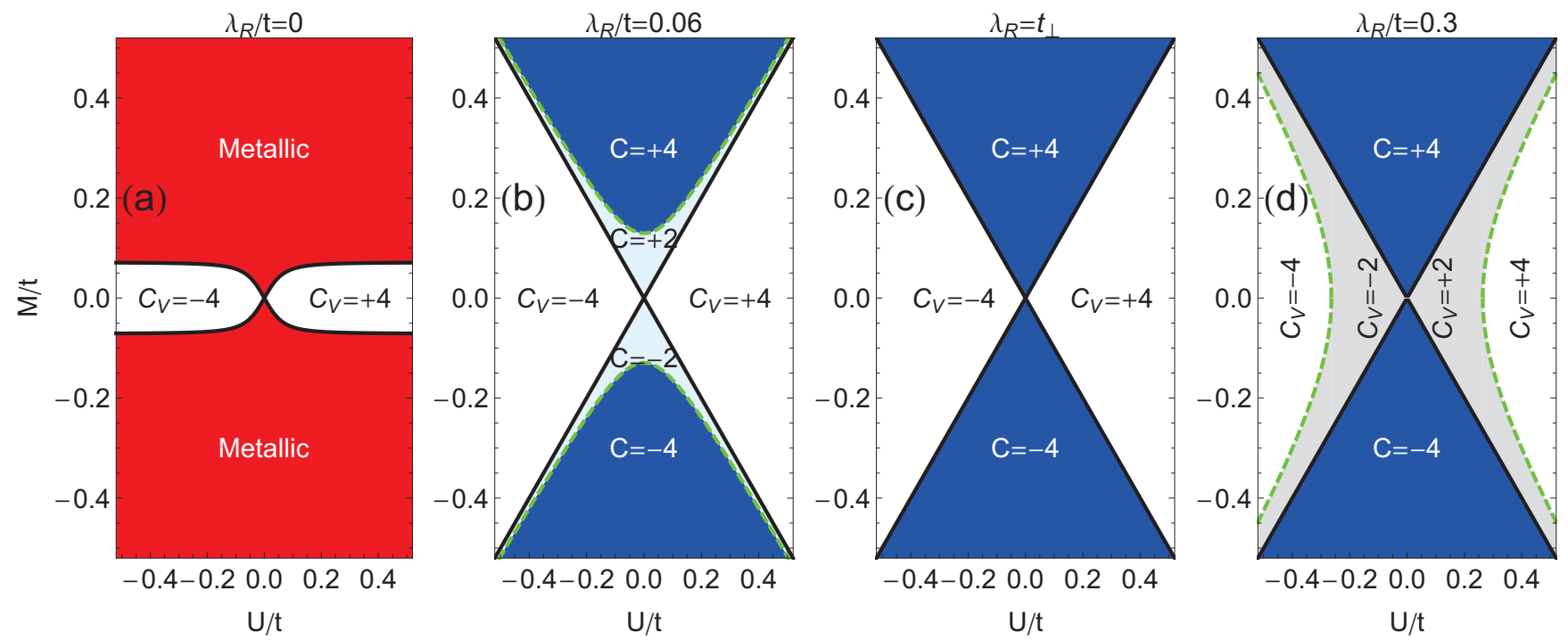

FIG. 15. (Color online) Phase diagram of bilayer graphene in the $(M, U)$ plane at different fixed Rashba spin-orbit couplings $\lambda_{R}$. Solid lines represent the phase boundary $U= \pm M$, which separate the quantum valley-Hall region and the quantum anomalous-Hall region. The dashed lines are given by Eqs. (28)-(29), separating the two different quantum anomalous Hall phases [see panel (a)] or the two different quantum valley-Hall phases [see panel (c)]. (a) $\lambda_{R}<t_{\perp}$. the quantum valley-Hall phase is characterized by $\mathcal{C}_{v}=4 \operatorname{sgn}(U)$ (in white), while the quantum anomalous-Hall region comprises two different phases represented by $C=4 \operatorname{sgn}(M)$ (in blue/dark) and $C=2 \operatorname{sgn}(M)$ (in gray). (b) $\lambda_{R}=t_{\perp}$. There is no sub-phase in each region. The quantum valley-Hall phase and quantum anomalous-Hall phase are respectively characterized by $\mathcal{C}_{v}=4 \operatorname{sgn}(U)($ in white) and $\mathcal{C}=4 \operatorname{sgn}(M)$ (in blue/dark). (c) $\lambda_{R}>t_{\perp}$. The quantum anomalous-Hall region has only one phase with Chern number being $\mathcal{C}=4 \operatorname{sgn}(M)$ (in blue/dark), while the quantum valley-Hall region includes two different phases with $\mathcal{C}_{v}=4 \operatorname{sgn}(U)$ (in white) and $\mathcal{C}_{v}=2 \operatorname{sgn}(U)$ (in gray).
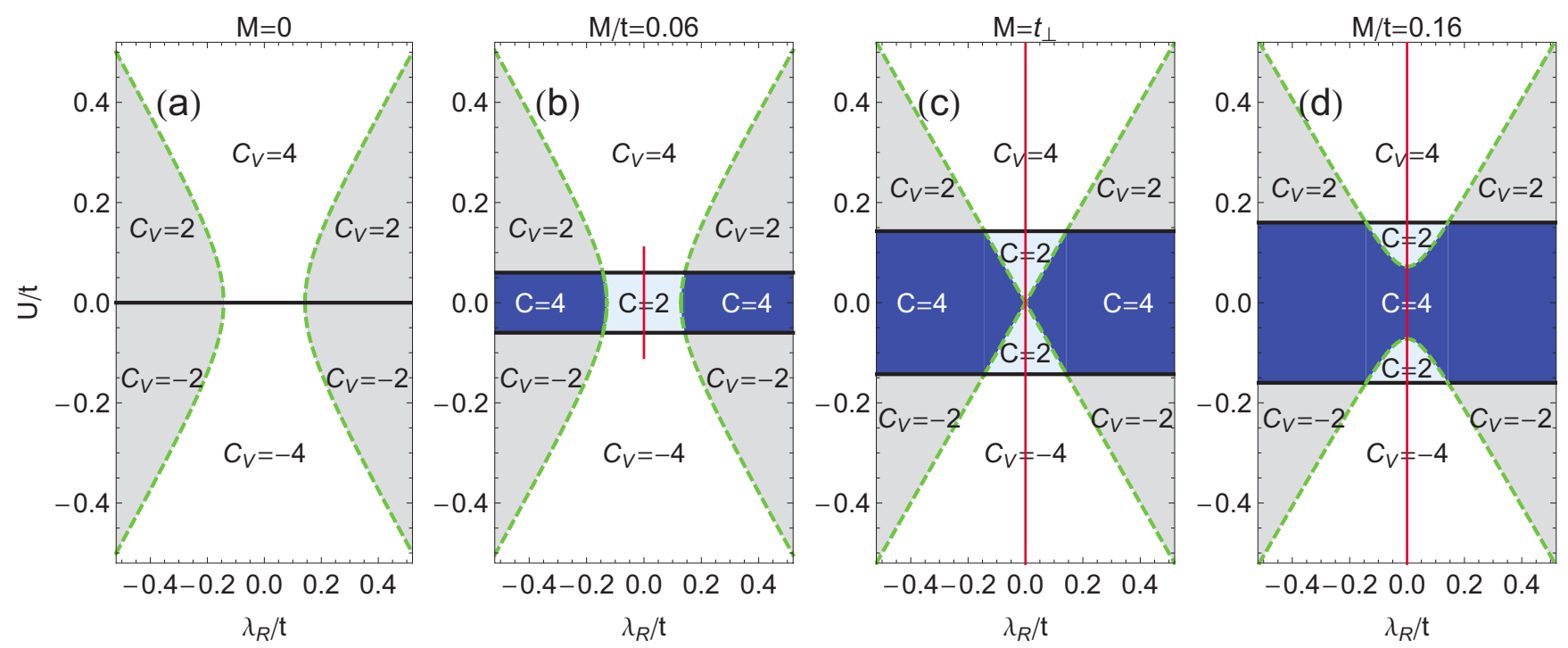

FIG. 16. (Color online) Phase diagram of bilayer graphene in the $\left(U, \lambda_{R}\right)$ plane at different fixed exchange fields $M$. Solid lines represent the phase boundary $U= \pm M$, which separate the quantum valley-Hall region and the quantum anomalousHall region. The dashed lines represent phase boundary separating two different sub-phases in the same region, given by $U^{2}+t_{\perp}^{2}-M^{2}-\lambda_{R}^{2}=0$. (a) $M=0$. This is just a profile along $t_{\perp} / t=0.1428$ in Fig. 2 to compare with panels (b) and (c). Red/dark regions are $Z_{2}$ topological insulator phase with $Z_{2}=1$ and $\mathcal{C}_{v}=2 \operatorname{sgn}(U)$. The white region is the $\mathcal{C}_{v}=4 \operatorname{sgn}(U)$ quantum valley-Hall phase. (b) $M<t_{\perp}$. Inside the region of $|U|<|M|$, quantum anomalous-Hall phases emerge. For larger $\lambda_{R}$, the Chern number is $\mathcal{C}=4$, while for small $\lambda_{R}$ approaching zero, it becomes $\mathcal{C}=2$. Outside the region of $|U|<|M|$, the valley-Chern numbers remain the same as those without exchange field as shown in panel (a). (c) $M=t_{\perp}$. The phase boundary becomes linear $U^{2}-\lambda_{R}^{2}=0$, which serves as a critical point changing the topology of the phase boundary. (d) $M>t_{\perp}$. The essential physics remains similar to that in panel (b). 
which gives rise to the phase transition condition

$$
\frac{4}{t_{\perp}^{2}}=\frac{1}{M^{2}}-\frac{1}{U^{2}} .
$$

In Fig. 14, we provide a vivid three-dimensional (3D) plot of the phase diagram in the $\left(M, \lambda_{R}, U\right)$ space. For clarity, we do not label each phase, but will distinguish them in the subsequent $2 \mathrm{D}$ phase diagrams in detail. One can observe that the whole $3 \mathrm{D}$ space is divided by a set of mutual vertical planes and a uniparted hyperboloid determined by Eqs. (28) and (29), respectively. It is noteworthy that the plane of $\lambda_{R}=0$ is distinct from other phase boundaries, i.e., the region labeled as red is a metallic phase. Below, we will explain the phase diagram by considering some representative regions.

Figure 15 exhibits the phase diagrams in the $(U, M)$ plane at four different fixed Rashba spin-orbit couplings: (a) $\lambda_{R}=0$, (b) $\lambda_{R}<t_{\perp}$, (c) $\lambda_{R}=t_{\perp}$ and (d) $\lambda_{R}>t_{\perp}$. In panel (a), one observes that for small $U$ the phase boundary is nearly linear to divide the metallic phase and quantum valley-Hall phase with valley Chern number $\mathcal{C}_{v}=4 \operatorname{sgn}(U)$, while for larger $U$ the phase phase boundary becomes a constant. As can be seen from other three graphs in (b)-(d), the fundamental division of the parameter space into QVHI phase and quantum anomalous-Hall phase are separated by the solid lines given by $U= \pm M$. In our calculation, the total Chern number is defined by $\mathcal{C}=\mathcal{C}_{K}+\mathcal{C}_{K^{\prime}}$. In Fig. 15), the valley Chern number in the QVHI phase is $\mathcal{C}_{v}=4 \operatorname{sgn}(U)$ in the white regime, while the quantum anomalous-Hall region comprises two different phases of matter with Chern numbers being $\mathcal{C}=2 \operatorname{sgn}(M)$ and $\mathcal{C}=4 \operatorname{sgn}(M)$ denoted in gray and blue, respectively. When $\lambda_{R}=t_{\perp}$, the phase boundary is only determined by $U= \pm M$, which is the same as we discussed in Ref. [13]. For a larger $\lambda_{R}$ as plotted in Fig. 15(d), the Chern number in the quantum anomalous-Hall phase is $\mathcal{C}=4 \operatorname{sgn}(M)$, while the QVHI region includes two different phases characterized by valley Chern numbers $\mathcal{C}_{v}=2 \operatorname{sgn}(U)$ and $\mathcal{C}_{v}=4 \operatorname{sgn}(U)$, represented in gray and white. It is interesting to point out that at fixed $M=0$ in the gray regime, it is both a $Z_{2}$ TI and a $\mathcal{C}_{v}=2 \operatorname{sgn}(U)$ QVHI.

In the above phase diagram, it is not obvious how the $Z_{2}$ TI phase is affected by the presence of exchange field. In Fig. 16, we plot the phase diagram in the $\left(U, \lambda_{R}\right)$ plane at four fixed exchange fields: (a) $M=0$; (b) $M<t_{\perp}$; (c) $M=t_{\perp}$; and (d) $M>t_{\perp}$. Figure [16(a) shows the phase diagram in the absence of exchange field, which is the profile of $t_{\perp}=0.1428 t$ in Fig. 2. We use gray and white colors to denote the $Z_{2}$ TI phase and conventional QVHI phase, respectively. When the exchange field is turned on, in Figs. 16(b)-(d), one finds that two different quantum anomalous Hall phases with Chern numbers $\mathcal{C}=2,4$ are induced when $U<M$ at nonzero Rashba effect. It is noteworthy that at finite $M$ the $Z_{2}$ TI phase vanishes due to the time-reversal symmetry breaking, but the valley Chern number remains quantized $\mathcal{C}_{v}=2 \operatorname{sgn}(U)$ when $|U|>M$. From these three graphs, one observes that the

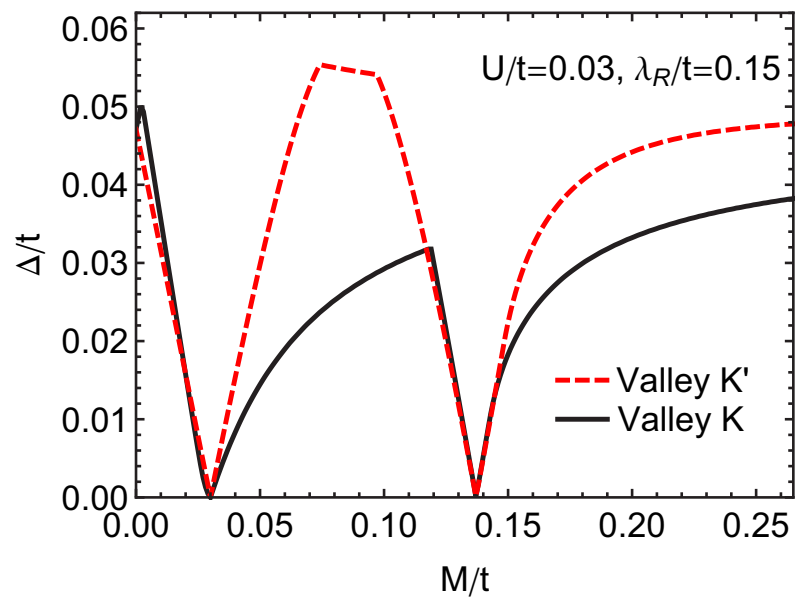

FIG. 17. (Color online) Bulk band gaps around valleys $K$ and $K^{\prime}$ as a function of the exchange field $M$ at fixed $U=0.03 t$ and $\lambda_{R}=0.15 t$. Here, the interlayer hopping is set to be $t_{\perp}=0.1428 t$. Solid and dashed lines represent the bulk band gap around $K$ and $K^{\prime}$, respectively. It can be clearly seen that the two bulk gaps are unequal in general, except for the two critical points where the bulk gaps are completely closed. Consistent with the phase-diagram in Fig. 15](c), it is a $\mathcal{C}_{v}=4$ quantum valley-Hall insulator for small $M$ before the first bulk gap closing; when $M$ is located in the interval between two bulk gap closing points, the system enters into a $\mathcal{C}_{v}=2$ quantum valley-Hall phase; for even larger $M$ exceeding the second critical point, it goes into a $\mathcal{C}=4$ quantum anomalousHall phase.

phase boundary labeled by the dashed lines are governed by Eq. (29), which reduces to the phase boundary equation Eq. (4) in the limit of $M=0$. This phase boundary indicates a continuity with and without exchange field. One also observes that with increasing exchange field, the topology of the phase boundary in dashed line changes at $M=t_{\perp}$. For zero Rashba SOC, when the exchange field is small, it closes the bulk band gap induced by small potential difference [see the vertical red line in panel (b)], driving the QVHI phase into a metallic phase; when the exchange field is large enough, the bulk gap from any potential difference is closed, giving rise to a complete metallic phase [see the red lines in panels (c) and (d)].

It is important to state that so far there are only a few papers 32,33 that report tunable Chern numbers in a quantum anomalous Hall system. From the above analysis, it is clear that our system provides another platform that hosts quantum anomalous Hall phases with different Chern numbers. The above phase diagrams are summarized concisely in Table I, which gives a complete classification of all possible topological phases in the gated bilayer graphene with Rashba spin-orbit coupling and exchange field, shown together with the necessary conditions for a particular phase to occur.

Another interesting feature in our system is that when all the three parameters $U, M$ and $\lambda_{R}$ are nonzero, the bulk gaps at valleys $K$ and $K^{\prime}$ have different responses. As an example, in Fig. 17 we present the results for the 
TABLE I. Summary of different topological phases in bilayer graphene in the presence of interlayer potential difference $U$, Rashba spin-orbit coupling $\lambda_{R}$, and exchange field $M$. They can be divided into two categories: quantum valley-Hall insulator (QVHI) and quantum anomalous-Hall insulator (QAHI). Note that the $Z_{2}$ TI also belongs to the QVHI phase.

\begin{tabular}{c|c|c|c|c}
\hline \hline & & $0<\left|\lambda_{R}\right|<t_{\perp}$ & $\left|\lambda_{R}\right|=t_{\perp}$ & $\left|\lambda_{R}\right|>t_{\perp}$ \\
\hline \multirow{2}{*}{$\mathrm{QVHHI}$} & $M=0$ & \multirow{2}{*}{$\mathcal{C}_{v}=4 \operatorname{sgn}(U)$} & - & $U^{2}>\lambda_{R}^{2}-t_{\perp}^{2}: \mathcal{C}_{v}=4 \operatorname{sgn}(U)$ \\
\cline { 5 - 5 } & \multirow{2}{*}{$0<M^{2}<U^{2}$} & $U^{2}<\lambda_{R}^{2}-t_{\perp}^{2}: \mathcal{C}_{v}=2 \operatorname{sgn}(U)$ and $Z_{2}=1$ \\
\hline \multirow{2}{*}{$\mathrm{QAHI}$} & \multirow{2}{*}{$U^{2}<M^{2}=4 \operatorname{sgn}(U)$} & $M^{2}>U^{2}+t_{\perp}^{2}-\lambda_{R}^{2}: \mathcal{C}=4 \operatorname{sgn}(M)$ & & $M^{2}<U^{2}+t_{\perp}^{2}-\lambda_{R}^{2}: \mathcal{C}_{v}=4 \operatorname{sgn}(U)$ \\
& & $M^{2}<U^{2}+t_{\perp}^{2}-\lambda_{R}^{2}: \mathcal{C}=2 \operatorname{sgn}(M)$ & $\mathcal{C}=4 \operatorname{sgn}(M)$ \\
\hline \hline
\end{tabular}

bulk band gaps as a function of the exchange field $M$ at fixed interlayer potential difference $U=0.03 t$ and Rashba spin-orbit coupling $\lambda_{R}=0.15 t$. It is clearly seen that as long as the exchange field term is turned on, the bulk gap amplitudes between $\mathrm{K}$ (solid line) and K' (dashed line) become unequal. However, even though the bulk gaps around the two valleys evolve quite differently, they close simultaneously. Again, the critical values of $M$ at the closing points agree very well with the analytic expression we derived in Eqs. (28)-(29).

\section{SUMMARY}

We have derived low-energy Hamiltonian descriptions for the TI phase and the QVHI phase in $A B$-stacked bilayer graphene with interlayer potential $U$ and Rashba spin-orbit coupling $\lambda_{R}$. We have explored the cases when the bilayer graphene has an $A A$-stacking or has different Rashba spin-orbit coupling strengths in the top and bottom layers. We showed that a $Z_{2}$ TI state can only be realized in the $A B$-stacked but not the $A A$-stacked bilayer graphene. To induce a strong enough Rashba spinorbit coupling in bilayer graphene, e.g., by heavy metal dopants or a substrate, different Rashba spin-orbit coupling strengths in the top and bottom layers $\lambda_{1} \neq \lambda_{2}$ could arise. We find that the TI phase can be realized as long as $\lambda_{1} \lambda_{2}>t_{\perp}^{2}$ for small interlayer potential difference. When the time-reversal symmetry is broken by an exchange field $M$, additional topological phases can be induced. We find that the QVHI phase and quantum anomalous-Hall phase are divided by $U= \pm M$. When $\lambda_{R} \neq t_{\perp}$, there exists another topological phase boundary determined by $U^{2}+t_{\perp}^{2}-M^{2}-\lambda_{R}^{2}=0$. For fixed $\lambda_{R}$, when $\lambda_{R}<t_{\perp}$, the quantum anomalous-Hall phase contains two different regions characterized by the Chern numbers of $C=2,4 \operatorname{sgn}(M)$; when $\lambda_{R}>t_{\perp}$, the QVHI phase separates into two regions characterized by the valley Chern numbers of $C_{v}=2,4 \operatorname{sgn}(U)$. Moreover, we find that when any two of the three parameters (i.e. interlayer potential difference, Rashba spin-orbit coupling, and the exchange field) are considered, the bulk band gaps at $K$ and $K^{\prime}$ are equal. However, if all three terms are present, the bulk gaps at $K$ and $K^{\prime}$ become different except at the topological phase transition points. It is noteworthy that in multilayer graphene, a bulk band gap opens in the presence of an external electric field. This makes multilayer graphene a good candidate to explore more interesting topological phases $\underline{\underline{34}}$

Acknowledgements. This work was financially supported by Welch Foundation (F-1255), DOE (DE-FG0302ER45958, Division of Materials Science and Engineering), the MOST Project of China (2012CB921300), and NSFC (91121004). H.J. was supported by the CPSF (20100480147 and 201104030). Y.Y. was supported by the NSF of China (10974231 and 11174337) and the MOST Project of China (2011CBA00100).

\section{A. Appendix}

In the following discussion we set $\lambda_{R}$ and $t_{\perp}$ as fixed, and allow $U$ to slightly deviate from the phase transition point $U_{0}$, i.e., $U=U_{0} \pm \Delta$, where $U_{0}=\sqrt{\lambda_{R}^{2}-t_{\perp}^{2}}$ and \pm correspond respectively to the quantum valley-Hall and topological insulator phases.

As stated in the main text, when the topological phase transition occurs, the bulk band gap closes at the exact Dirac points $K / K^{\prime}$. At the critical $U_{0}$, the system Hamiltonian on the basis of $\left\{B_{1 \downarrow}, A_{2 \uparrow}, A_{1 \uparrow}, A_{2 \downarrow}, B_{2 \uparrow}, B_{2 \downarrow}, B_{1 \uparrow}, A_{1 \downarrow}\right\}$ can be expressed as

$$
H\left(U_{0}\right)=\left[\begin{array}{cccccccc}
U_{0} & 0 & 0 & 0 & 0 & 0 & 0 & 0 \\
0 & -U_{0} & 0 & 0 & 0 & 0 & 0 & 0 \\
0 & 0 & U_{0} & 0 & t_{\perp} & 0 & 0 & 0 \\
0 & 0 & 0 & -U_{0} & -i \lambda_{R} & 0 & 0 & 0 \\
0 & 0 & t_{\perp} & i \lambda_{R} & -U_{0} & 0 & 0 & 0 \\
0 & 0 & 0 & 0 & 0 & -U_{0} & 0 & t_{\perp} \\
0 & 0 & 0 & 0 & 0 & 0 & U_{0} & i \lambda_{R} \\
0 & 0 & 0 & 0 & 0 & t_{\perp} & -i \lambda_{R} & U_{0}
\end{array}\right]
$$

Through a direct diagonalization, the eigenenergies are obtained as $\varepsilon=0,0, \pm U_{0}, \pm \varepsilon_{1}, \pm \varepsilon_{2}$, where $\varepsilon_{1}=-U_{0} / 2+$ $\sqrt{8 \lambda_{R}^{2}+U_{0}^{2}} / 2$ and $\varepsilon_{2}=-U_{0} / 2-\sqrt{8 \lambda_{R}^{2}+U_{0}^{2}} / 2$. The former four correspond to the low-energy part, while the 
latter four correspond to the high-energy part. Based on our analysis of the Berry curvature from the tight-binding model, the valley-Chern number arises only from the lowenergy bands and the high-energy bands contribute zero.

For the diagonal block Hamiltonian of $h_{1}\left(U_{0}\right)=$ $\left[\begin{array}{ccc}U_{0} & 0 & t_{\perp} \\ 0 & -U_{0} & -i \lambda_{R} \\ t_{\perp} & i \lambda_{R} & -U_{0}\end{array}\right]$, its unitary transformation matrix is

$$
\begin{aligned}
\mathbf{V}_{1}= & {\left[\begin{array}{lll}
v_{11} & v_{12} & v_{13} \\
v_{21} & v_{22} & v_{23} \\
v_{31} & v_{32} & v_{33}
\end{array}\right] } \\
= & {\left[\begin{array}{ccc}
\frac{t_{\perp}}{\sqrt{2} \lambda_{R}} & 1 / \alpha_{2} & 1 / \alpha_{3} \\
\frac{i}{\sqrt{2}} & \frac{i \lambda_{R}\left(U_{0}-\varepsilon_{1}\right)}{\alpha_{2} t_{\perp}\left(U_{0}+\varepsilon_{1}\right)} & \frac{i \lambda_{R}\left(U_{0}-\varepsilon_{2}\right)}{\alpha_{3} t_{\perp}\left(U_{0}+\varepsilon_{2}\right)} \\
\frac{-U_{0}}{\sqrt{2} \lambda_{R}} & \frac{\varepsilon_{1}-U_{0}}{\alpha_{2} t_{\perp}} & \frac{\varepsilon_{2}-U_{0}}{\alpha_{3} t_{\perp}}
\end{array}\right], }
\end{aligned}
$$

which can diagonalize $h_{1}\left(U_{0}\right)$ to be

$$
h_{1}^{\prime}\left(U_{0}\right)=\mathbf{V}_{1}^{\dagger} h_{1}\left(U_{0}\right) \mathbf{V}_{1}=\left[\begin{array}{ccc}
0 & 0 & 0 \\
0 & \varepsilon_{1} & 0 \\
0 & 0 & \varepsilon_{2}
\end{array}\right] \text {. }
$$

$\alpha_{2}$ and $\alpha_{3}$ are respectively

$$
\begin{aligned}
& \alpha_{2}=\sqrt{1+\left[\frac{\lambda_{\mathrm{R}}\left(U_{0}-\varepsilon_{1}\right)}{\left(U_{0}+\varepsilon_{1}\right) t_{\perp}}\right]^{2}+\left[\frac{\varepsilon_{1}-U_{0}}{t_{\perp}}\right]^{2}}, \\
& \alpha_{3}=\sqrt{1+\left[\frac{\lambda_{\mathrm{R}}\left(U_{0}-\varepsilon_{2}\right)}{\left(U_{0}+\varepsilon_{2}\right) t_{\perp}}\right]^{2}+\left[\frac{\varepsilon_{2}-U_{0}}{t_{\perp}}\right]^{2}} .
\end{aligned}
$$

For the other diagonal block Hamiltonian of $h_{2}\left(U_{0}\right)=$ $\left[\begin{array}{ccc}-U_{0} & 0 & t_{\perp} \\ 0 & U_{0} & i \lambda_{R} \\ t_{\perp} & -i \lambda_{R} & U_{0}\end{array}\right]$, its unitary transformation matrix is written as

$$
\mathbf{V}_{2}=\left[\begin{array}{ccc}
v_{11} & v_{12} & v_{13} \\
-v_{21} & -v_{22} & -v_{23} \\
-v_{31} & -v_{32} & -v_{33}
\end{array}\right]
$$

which leads to

$$
h_{2}^{\prime}\left(U_{0}\right)=\mathbf{V}_{2}^{\dagger} h_{2}\left(U_{0}\right) \mathbf{V}_{2}=\left[\begin{array}{ccc}
0 & 0 & 0 \\
0 & -\varepsilon_{1} & 0 \\
0 & 0 & -\varepsilon_{2}
\end{array}\right] .
$$

In order to arrange the eigenenergies to be low and high energy parts, the basis should be reordered to be: $\left\{A_{1 \uparrow}, B_{2 \downarrow}, B_{1 \downarrow}, A_{2 \uparrow}, A_{2 \downarrow}, B_{2 \uparrow}, B_{1 \uparrow}, A_{1 \downarrow}\right\}$. The corresponding full unitary transformation matrix becomes:

$$
\mathbf{V}=\left[\begin{array}{cccccccc}
0 & 0 & 1 & 0 & 0 & 0 & 0 & 0 \\
0 & 0 & 0 & 1 & 0 & 0 & 0 & 0 \\
v_{11} & 0 & 0 & 0 & v_{12} & v_{13} & 0 & 0 \\
v_{21} & 0 & 0 & 0 & v_{22} & v_{23} & 0 & 0 \\
v_{31} & 0 & 0 & 0 & v_{32} & v_{33} & 0 & 0 \\
0 & v_{11} & 0 & 0 & 0 & 0 & v_{12} & v_{13} \\
0 & -v_{21} & 0 & 0 & 0 & 0 & -v_{22} & -v_{23} \\
0 & -v_{31} & 0 & 0 & 0 & 0 & -v_{32} & -v_{33}
\end{array}\right] .
$$

When the interlayer potential difference is slightly deviated from $U_{0}$, the Hamiltonian is written as:

$$
H(U)=H\left(U_{0}\right)+H_{\Delta}+H_{\boldsymbol{k}},
$$

where

$$
H_{\Delta}=\operatorname{Diag}\{\Delta,-\Delta, \Delta,-\Delta,-\Delta,-\Delta, \Delta, \Delta\} .
$$

and

$$
H_{\boldsymbol{k}}=\left[\begin{array}{rccccccc}
0 & 0 & 0 & 0 & 0 & 0 & 0 & v k_{+} \\
0 & 0 & 0 & 0 & v k_{-} & 0 & 0 & 0 \\
0 & 0 & 0 & 0 & 0 & 0 & v k_{-} & 0 \\
0 & 0 & 0 & 0 & 0 & v k_{-} & 0 & 0 \\
0 & v k_{+} & 0 & 0 & 0 & 0 & 0 & 0 \\
0 & 0 & 0 & v k_{+} & 0 & 0 & 0 & 0 \\
0 & 0 & v k_{+} & 0 & 0 & 0 & 0 & 0 \\
v k_{-} & 0 & 0 & 0 & 0 & 0 & 0 & 0
\end{array}\right] .
$$

By performing a unitary transformation, the Hamiltonian of $H(U)$ becomes 


$$
\begin{aligned}
H^{\prime}(U)= & \mathbf{V}^{\dagger} H(U) \mathbf{V} \\
= & {\left[\begin{array}{cccccccc}
\left(2 v_{11}^{2}-1\right) \Delta & \gamma_{1} v k_{-} & 0 & v_{31} v k_{+} & 2 v_{11} v_{12} \Delta & 2 v_{11} v_{13} \Delta & \gamma_{2} v k_{-} & \gamma_{3} v k_{-} \\
-\gamma_{1} v k_{+} & \left(1-2 v_{11}^{2}\right) \Delta & -v_{31} v k_{-} & 0 & -\gamma_{2} v k_{+} & -\gamma_{3} v k_{+} & -2 v_{11} v_{12} \Delta & -2 v_{11} v_{13} \Delta \\
0 & -v_{31} v k_{+} & \Delta+U_{0} & 0 & 0 & 0 & -v_{32} v k_{+} & -v_{33} v k_{+} \\
v_{31} v k_{-} & 0 & 0 & -\Delta-U_{0} & v_{32} v k_{-} & v_{33} v k_{-} & 0 & 0 \\
2 v_{11} v_{12} \Delta & \gamma_{2} v k_{-} & 0 & v_{32} v k_{+} & \gamma_{4} * \Delta+\varepsilon_{1} & \gamma_{5} \Delta & -2 v_{12} v_{22} v k_{-} & \gamma_{8} v k_{-} \\
2 v_{11} v_{13} \Delta & \gamma_{3} v k_{-} & 0 & v_{33} v k_{+} & \gamma_{5} \Delta & \gamma_{6} \Delta+\varepsilon_{2} & \gamma_{8} v k_{-} & -2 v_{13} v_{23} v k_{-} \\
-\gamma_{2} v k_{+} & -2 v_{11} v_{12} \Delta & -v_{32} v k_{-} & 0 & 2 v_{12} v_{22} v k_{+} & -\gamma_{8} v k_{+} & -\gamma_{4} * \Delta-\varepsilon_{1} & \gamma_{7} \Delta \\
-\gamma_{3} v k_{+} & -2 v_{11} v_{13} \Delta & -v_{33} v k_{-} & 0 & -\gamma_{8} v k_{+} & 2 v_{13} v_{23} v k_{+} & \gamma_{7} \Delta & -\gamma_{6} \Delta-\varepsilon_{2}
\end{array}\right] } \\
= & {\left[\begin{array}{ccccccc}
H_{P} \\
T \\
T^{\dagger}
\end{array}\right], }
\end{aligned}
$$

where $\gamma_{1}=-2 v_{11} v_{21}=-i t_{\perp} / \lambda_{R}, \gamma_{2}=-\left(v_{11} v_{22}+\right.$ $\left.v_{12} v_{21}\right), \gamma_{3}=-\left(v_{11} v_{23}+v_{13} v_{21}\right), \gamma_{4}=v_{12}^{2}+v_{22}^{2}-v_{32}^{2}$, $\gamma_{5}=v_{12} v_{13}+v_{22} v_{23}-v_{32} v_{33}, \gamma_{6}=v_{13}^{2}+v_{23}^{2}-v_{33}^{2}$, $\gamma_{7}=v_{32} v_{33}-v_{22} v_{23}-v_{12} v_{13}$, and $\gamma_{8}=-\left(v_{12} v_{23}+v_{13} v_{22}\right)$. Since both $\Delta$ and $k_{+} / h_{-}$are extremely small, the effective Hamiltonian can be simplified to be

$$
H_{e f f}=H_{P}-T H_{Q}^{-1} T^{\dagger}
$$

1 M. Z. Hasan and C. L. Kane, Rev. Mod. Phys. 82, 3045 (2010); X.-L. Qi and S.-C. Zhang, Rev. Mod. Phys. 83, 1057 (2011).

2 C. L. Kane, and E. J. Mele, Phys. Rev. Lett. 95, 146802 (2005); Phys. Rev. Lett. 95, 226801 (2005).

3 B. A. Bernevig, T. L. Hughes, and S.-C. Zhang, Science 314, 1757 (2006).

${ }^{4}$ H. Min et al., Phys. Rev. B 74, 165310 (2006); Y. G. Yao et al., Phys. Rev. B 75, 041401(R) (2007); M. Gmitra et al., Phys. Rev. B 80, 235431 (2009).

5 M. Koenig et al., Science 318, 766 (2007).

${ }^{6}$ I. Knez, R.-R. Du, G. Sullivan, Phys. Rev. Lett. 107, 136603 (2011).

7 C. Weeks, J. Hu, J. Alicea, M. Franz, and R. Q. Wu, Phys. Rev. X 1, 021001 (2011).

${ }^{8}$ H. B. Zhang, C. Lazo, S. Blügel1, S. Heinze, and Y. Mokrousov, Phys. Rev. Lett. 108, 056802 (2012).

9 Z. H. Qiao, S. A. Yang, W. X. Feng, W.-K. Tse, J. Ding, Y. G. Yao, J. Wang, and Q. Niu, Phys. Rew. B 82, 161414(R)
Explicitly, $H_{P}$ can be written as:

$$
H_{P}=\left[\begin{array}{cccc}
\frac{-U_{0}^{2} \Delta}{\lambda_{R}^{2}} & \frac{-i t_{\perp} v k_{-}}{\lambda_{R}} & 0 & \frac{-U_{0} v k_{+}}{\sqrt{2} \lambda_{R}} \\
\frac{i t_{\perp} v k_{+}}{\lambda_{R}} & \frac{U_{0}^{2} \Delta}{\lambda_{R}^{2}} & \frac{U_{0} v k_{-}}{\sqrt{2} \lambda_{R}} & 0 \\
0 & \frac{U_{0} v k_{+}}{\sqrt{2} \lambda_{R}} & \Delta+U_{0} & 0 \\
\frac{-U_{0} v k_{-}}{\sqrt{2} \lambda_{R}} & 0 & 0 & -\Delta-U_{0}
\end{array}\right]
$$

For small $\Delta$ and $\boldsymbol{k}$, the higher energy block can be expressed as:

$$
H_{Q}=\left[\begin{array}{cccc}
\varepsilon_{1} & 0 & 0 & 0 \\
0 & \varepsilon_{2} & 0 & 0 \\
0 & 0 & -\varepsilon_{1} & 0 \\
0 & 0 & 0 & -\varepsilon_{2}
\end{array}\right]
$$

10 J. Ding, Z. H. Qiao, W. X. Feng, Y. G. Yao, and Q. Niu, Phys. Rev. B 84, 195444 (2011).

11 A. Varykhalov et al., Phys. Rev. Lett. 101, 157601 (2008); Y. S. Dedkov et al., Phys. Rev. Lett. 100, 107602 (2008); O. Rader et al., Phys. Rev. Lett. 102, 057602 (2009); A. Varykhalov and O. Rader, Phys. Rev. B 80, 035437 (2009).

12 Z. H. Qiao, W.-K. Tse, H. Jiang, Y. G. Yao, and Q. Niu. Phys. Rev. Lett. 107, 256801 (2011).

13 W.-K. Tse, Z. H. Qiao, Y. G. Yao, A. H. MacDonald, and Q. Niu, Phys. Rev. B 83, 155447 (2011).

14 Z. H. Qiao, H. Jiang, X. Li, Y. G. Yao, and Q. Niu, Phys. Rev. B 85, 115439 (2012).

15 L. Sheng, D. N. Sheng, C. S. Ting, and F.D.M. Haldane, Phys. Rev. Lett. 95, 136602 (2005); D. N. Sheng, Z. Y. Weng, L. Sheng, and F.D.M. Haldane, Phys. Rev. Lett. 97, 036808 (2006).

16 The lattice unit vectors are the same as those in Ref. [14].

17 For details of the expression of each term, one can refer to 
Ref. 14].

18 This is reasonable, because based on our numerical results the high-energy valence bands have respectively opposite Berry curvatures making no contribution to the total Berry curvatures.

19 D. Xiao, W. Yao, and Q. Niu, Phys. Rev. Lett. 99, 236809 (2007).

20 The corresponding band structures from the model Hamiltonian at $K^{\prime}$ are exactly the same as those from valley $K$.

21 R. Bistritzer and A.H. MacDonald, Proc. Natl. Acad. Sci. 108, 12233 (2011).

22 R. Bistritzer and A.H. MacDonald, Phys. Rev. B 84, 035440 (2011)

${ }^{23}$ D. S. Lee, C. Riedl, T. Beringer, A. H. Castro Neto, K. von Klitzing, U. Starke, and J. H. SmetPhys. Rev. Lett. 107, 216602 (2011).

24 E. J. Mele, Phys. Rev. B 81, 161405(R) (2010)

${ }^{25}$ R. de Gail, M. O. Goerbig, F. Guinea, G. Montambaux, A. H. Castro Neto, arXiv:1103.3172 (unpublished).

${ }^{26}$ L. Fu and C. L. Kane, Phys. Rev. B 74, 195312 (2006); D.
Xiao, Y. G. Yao, W. X. Feng, J. Wen, W. G. Zhu, X.-Q. Chen, G. M. Stocks, and Z. Y. Zhang, Phys. Rev. Lett. 105, 096404 (2010); T. Fukui, and Y. Hatsugai, J. Phys. Soc. Jpn. 76, 053702 (2007); A. M. Essin, and J. E. Moore, Phys. Rev. B 76, 165307 (2007).

27 F. D. M. Haldane, Phys. Rev. Lett. 61, 2015 (1988).

28 C.-X. Liu, X.-L. Qi, X. Dai, Z. Fang, and S.-C. Zhang, Phys. Rev. Lett. 101, 146802 (2008).

29 M. Onoda and N. Nagaosa, Phys. Rev. Lett. 90, 206601 (2003).

30 C. Wu, Phys. Rev. Lett. 101, 186807 (2008); Y. P. Zhang and C. W. Zhang, Phys. Rev. B 84, 085123 (2011).

31 R. Yu, W. Zhang, H.-J. Zhang, S.-C. Zhang, X. Dai, and Z. Fang, Science 329, 61 (2010).

32 T.-W. Chen, Z.-R. Xiao, D.-W. Chiou, and G.-Y. Guo, Phys. Rev. B 84, 165453 (2011).

33 H. Jiang, Z. H. Qiao, H. W. Liu, and Q. Niu, Phys. Rev. B 85, 045445 (2012).

34 X. Li, Z. H. Qiao, J. Jung, and Q. Niu, Phys. Rew. B 85, 201404(R) (2012). 